\title{
TESS and HARPS reveal two sub-Neptunes around TOI 1062
}

\author{
J. F. Otegi ${ }^{1,2}$, F. Bouchy ${ }^{1}$, R. Helled ${ }^{2}$, D. J. Armstrong ${ }^{19,20}$, M. Stalport ${ }^{1}$, A. Psaridi ${ }^{1}$, J.-B. Delisle ${ }^{1}$, K.G. Stassun ${ }^{13}$, \\ E. Delgado-Mena ${ }^{17}$, N. C. Santos ${ }^{17,18}$, N. C. Hara ${ }^{1}$, K. Collins ${ }^{7}$, S. Gandhi ${ }^{19,20}$, C. Dorn ${ }^{2}$, M. Brogi ${ }^{19,20}$, \\ M. Fridlund ${ }^{25,26}$, H. P. Osborn ${ }^{23,24}$, S. Hoyer ${ }^{27}$, S. Udry ${ }^{1}$, S. Hojjatpanah ${ }^{17,18}$, L. D. Nielsen ${ }^{1}$, X. Dumusque ${ }^{1}$, \\ V. Adibekyan ${ }^{17,18}$, D. Conti ${ }^{8}$, R. Schwarz ${ }^{9}$, G. Wang ${ }^{10}$, P. Figueira ${ }^{17,28}$, J. Lillo-Box ${ }^{3}$, A. Hadjigeorghiou ${ }^{19,20}$, \\ D. Bayliss ${ }^{19,20}$, P. A. Strøm ${ }^{19,20}$, S. G. Sousa ${ }^{17}$, D. Barrado ${ }^{3}$, A. Osborn ${ }^{19,20}$, S. C. C. Barros ${ }^{17,18}$, D. J. A. Brown ${ }^{19,20}$, \\ J. D. Eastman ${ }^{7}$, D. R. Ciardi $^{16}$, A. Vanderburg ${ }^{15}$, R. F. Goeke ${ }^{4}$, N. M. Guerrero ${ }^{4}$, P. T. Boyd ${ }^{14}$, D. A. Caldwell ${ }^{12,21}$, \\ C. E. Henze ${ }^{12}$, B. McLean ${ }^{22}$, G. Ricker ${ }^{4}$, R. Vanderspek ${ }^{4}$, D. W. Latham ${ }^{7}$, S. Seager ${ }^{4,5,6}$, J. Winn ${ }^{11}$, and J. M. Jenkins ${ }^{12}$
}

(Affiliations can be found after the references)

Received 27 December 2020 / Accepted 5 May 2021

\begin{abstract}
The Transiting Exoplanet Survey Satellite (TESS) mission was designed to perform an all-sky search of planets around bright and nearby stars. Here we report the discovery of two sub-Neptunes orbiting around TOI 1062 (TIC 299799658), a $V=10.25$ G9V star observed in the TESS Sectors 1, 13, 27, and 28. We use precise radial velocity observations from HARPS to confirm and characterize these two planets. TOI $1062 \mathrm{~b}$ has a radius of $2.265_{-0.091}^{+0.096} R_{\oplus}$, a mass of $10.15 \pm 0.8 M_{\oplus}$, and an orbital period of $4.1130 \pm 0.0015$ days. The second planet is not transiting, has a minimum mass of $9.78_{-1.18}^{+1.26} M_{\oplus}$ and is near the $2: 1$ mean motion resonance with the innermost planet with an orbital period of $7.972_{-0.024}^{+0.018}$ days. We performed a dynamical analysis to explore the proximity of the system to this resonance, and to attempt further constraining the orbital parameters. The transiting planet has a mean density of $4.85_{-0.74}^{+0.84} \mathrm{~g} \mathrm{~cm}^{-3}$ and an analysis of its internal structure reveals that it is expected to have a small volatile envelope accounting for $0.35 \%$ of the mass at most. The star's brightness and the proximity of the inner planet to what is know as the radius gap make it an interesting candidate for transmission spectroscopy, which could further constrain the composition and internal structure of TOI $1062 \mathrm{~b}$.
\end{abstract}

Key words. planets and satellites: general - planets and satellites: detection - planets and satellites: composition

\section{Introduction}

The Kepler mission was the first exoplanet mission to perform a large statistical survey of transiting exoplanets (Borucki et al. 2010; Howell et al. 2014); it impacted the field significantly with the detection of over 2300 exoplanets (see NASA Exoplanet Archive Akeson et al. 2013). Nevertheless, due to the faintness of the stars targeted by Kepler, only a small fraction are suitable for radial velocity (RV) follow-up. The Transiting Exoplanet Survey Satellite (TESS) has been designed to survey $85 \%$ of the sky for transiting exoplanets around bright, nearby stars (Ricker et al. 2015). It spent the first year of its mission searching for planets in the Ecliptic Southern Hemisphere. More than 2000 candidates have been detected and over 80 have been confirmed, expanding the number of small planets around cool stars (Guerrero 2020). $\mathrm{RV}$ follow-up programs have allowed us to quickly validate transiting planet candidates and to constrain their masses. Accurate measurements of the planetary mass and radius provide information on the planet's bulk density. However, a full characterization on the planetary composition and internal structure is extremely challenging due to the degenerate nature of this problem, where various compositions and interiors can lead to the same average density. Nevertheless, by studying the population of planets in this mass and size range, it is possible to better understand the dominating processes of planet formation and evolution in a statistical manner (Alibert et al. 2010, 2015).

A key signature in the planet population is the hot Neptune desert, which corresponds to a lack of Neptune-mass planets close to their host stars (Szabó \& Kiss 2011). The desert is likely to arise from a combination of photoevaporation and tidal disruption (Beaugé \& Nesvorný 2013; Mazeh et al. 2016). At small orbital distances planets are subject to intense radiation from the host stars, and Neptune-like planets are probably not massive enough to retain their gaseous envelopes. An important advantage of the all-sky nature of TESS resides in its ability to find the brightest examples of stars hosting rare sub-populations of planets, such as the exoplanets lying inside the desert. TESS has been able to find the first planets deep inside the desert, for example TOI-849b (Armstrong et al. 2020), a recently discovered remnant core of a giant planet, and LTT9779b (Jenkins et al. 2020), an ultra-hot Neptune. The NCORES HARPS large program is designed to further study planets that may have undergone substantial envelope loss. Some of the recently discovered planets under this program include the mentioned TOI-849b and three mini Neptunes TOI-125b, c, d (Nielsen et al. 2020).

Another relevant feature in the exoplanet population is a lack of planets with radii between $1.5 R_{\oplus}$ and $2 R_{\oplus}$ (Fulton et al. 2017) known as the radius valley. This suggests that there is a transition between the super-Earth and sub-Neptune populations (Owen \& Jackson 2012). This gap could be a result of stellar irradiation (Lopez \& Fortney 2013) or core-powered mass-loss (e.g., Ginzburg et al. 2018), which leads to atmospheric evaporation that strips the planet down to its core or, alternatively, the consequence of a gas-poor formation (e.g., Gupta \& Schlichting 2019). The observed features of the exoplanet populations are not well understood, and more exoplanet discoveries are crucial to reveal 
Table 1. Stellar parameters of TOI-1062.

\begin{tabular}{l}
\hline Parameter \\
\hline Identifying information \\
TOI \\
TIC ID \\
2MASS ID \\
Gaia ID
\end{tabular}

Astrometric parameters

RA (J2015.5, h:m:s)

Dec (J2015.5, h:m:s)

Parallax (mas)

Distance (pc)

TOI-1062
299799658
J02322848-7801256
4632865331094140928

TESS

TESS

2MASS

Gaia DR3

Photometric parameters

\begin{tabular}{lc}
\hline $\mathrm{B}$ & $11.02 \pm 0.07$ \\
$\mathrm{~V}$ & $10.25 \pm 0.01$ \\
$\mathrm{~T}$ & $9.48 \pm 0.01$ \\
$\mathrm{G}$ & $10.00 \pm 0.01$ \\
$\mathrm{~J}$ & $8.78 \pm 0.02$ \\
$\mathrm{H}$ & $8.41 \pm 0.03$ \\
$\mathrm{~K}$ & $8.30 \pm 0.03$ \\
$\mathrm{~W} 1$ & $8.257 \pm 0.02$ \\
$\mathrm{~W} 2$ & $8.322 \pm 0.02$ \\
$\mathrm{~W} 3$ & $8.256 \pm 0.02$ \\
$\mathrm{~W} 4$ & $8.35 \pm 0.19$ \\
$A_{\mathrm{V}}$ & $0.13 \pm 0.04$
\end{tabular}

\section{Abundances}

$[\mathrm{Fe} / \mathrm{H}](\mathrm{dex})$

$[\mathrm{O} / \mathrm{H}](\mathrm{dex})$

$[\mathrm{C} / \mathrm{H}](\mathrm{dex})$

$[\mathrm{Cu} / \mathrm{H}](\mathrm{dex})$

$[\mathrm{Zn} / \mathrm{H}](\mathrm{dex})$

$[\mathrm{Sr} / \mathrm{H}](\mathrm{dex})$

$[\mathrm{Y} / \mathrm{H}](\mathrm{dex})$

$[\mathrm{Zr} / \mathrm{H}](\mathrm{dex})$

$[\mathrm{Ba} / \mathrm{H}](\mathrm{dex})$

$[\mathrm{Ce} / \mathrm{H}](\mathrm{dex})$

$[\mathrm{Nd} / \mathrm{H}](\mathrm{dex})$

$[\mathrm{MgI} / \mathrm{H}](\mathrm{dex})$

$[\mathrm{AlI} / \mathrm{H}](\mathrm{dex})$

$[\mathrm{SiI} / \mathrm{H}](\mathrm{dex})$

$[\mathrm{CaI} / \mathrm{H}](\mathrm{dex})$

$[\mathrm{TiI} / \mathrm{H}](\mathrm{dex})$

$[\mathrm{CrI} / \mathrm{H}](\mathrm{dex})$

$[\mathrm{NiI} / \mathrm{H}](\mathrm{dex})$

$$
\begin{gathered}
0.14 \pm 0.04 \\
-0.14 \pm 0.16 \\
0.13 \pm 0.02 \\
0.25 \pm 0.04 \\
0.08 \pm 0.04 \\
0.17 \pm 0.08 \\
0.04 \pm 0.08 \\
0.20 \pm 0.06 \\
-0.02 \pm 0.04 \\
0.16 \pm 0.10 \\
0.10 \pm 0.06 \\
0.16 \pm 0.07 \\
0.25 \pm 0.04 \\
0.20 \pm 0.07 \\
0.10 \pm 0.08 \\
0.23 \pm 0.07 \\
0.17 \pm 0.07 \\
0.17 \pm 0.05
\end{gathered}
$$

\section{Bulk parameters}

\begin{tabular}{lcc}
\hline Mass $\left(M_{\odot}\right)$ & $0.94 \pm 0.02$ & Sect. 3 \\
Radius $\left(R_{\odot}\right)$ & $0.84 \pm 0.09$ & Sect. 3 \\
$T_{\text {eff }}(\mathrm{K})$ & $5328 \pm 56$ & Sect. 3 \\
$\log g\left(\mathrm{~cm} \mathrm{~s}^{-2}\right)$ & $4.55 \pm 0.09$ & Sect. 3 \\
Spectral type & G9V & Sect. 3 \\
$\rho\left(\mathrm{g} \mathrm{cm}^{-3}\right)$ & $2.22 \pm 0.10$ & Sect. 3 \\
$v \sin i\left(\mathrm{~km} \mathrm{~s}^{-1}\right)$ & $2.13 \pm 0.5$ & Sect. 3 \\
$P_{\text {rot }}(\mathrm{d})$ & $21.8 \pm 2.4$ & Sect. 3 \\
Age $(\mathrm{Gyrs})$ & $2.5 \pm 0.3$ & Sect. 3 \\
\hline
\end{tabular}

References. 2MASS (Skrutskie et al. 2006); Tycho (Høg et al. 2000); WISE (Wright et al. 2010); and Gaia (Gaia Collaboration 2018).

Tycho

TESS

Gaia

2MASS

2MASS

2MASS

WISE

WISE

WISE

WISE

Sect. 3

Sect. 3

Sect. 3

Sect. 3

Sect. 3

Sect. 3

Setc. 3

Sect. 3

Sect. 3

Sect. 3

Sect. 3

Sect. 3

Sect. 3

Sect. 3

Sect. 3

Sect. 3

Sect. 3

Sect. 3

Sect. 3 the overall picture of planets in the super-Earth and Neptune size and mass regimes.

In this paper we present the discovery and confirmation of two highly irradiated sub-Neptunes hosted by the TESS object of interest (TOI) 1062, a nearby $(d=82 \mathrm{pc})$ and bright $\left(V_{\text {mag }}=10.2\right)$ G9V star (see Table 1 for a full summary of the stellar properties). We also used intensive radial velocity followup observations with HARPS to confirm the planetary nature of the transit detected in the TESS data and precisely determine the properties of the planetary system. The paper is organized as follows: in Sect. 2 we present the data collected on the system to discover and validate the planets, in Sect. 3 we analyze the host star fundamental parameters, and in Sect. 4 we characterize and discuss the planets. Our conclusions are presented in Sect. 5.

\section{Observations}

\subsection{TESS photometry}

TESS observed TOI 1062 (TIC 299799658) in Sectors 1 and 13 during the first year, and during Sectors 27 and 28 of the extended mission, obtaining data from 25 July to 22 August 2018, from 19 June to 17 July 2018, and from 4 July to 26 August 2020. The two-minute cadence data were reduced with the Science Processing Operations Center (SPOC) pipeline (Jenkins et al. 2016) adapted from the pipeline for the Kepler mission at the NASA Ames Research Center in order to produce calibrated pixels and light curves. On 29 September 2018 a transit candidate was announced around TOI 1062. TOI 1062.01 is a planet candidate with a period of $4.11506 \pm 0.00002$ days, a transit depth of $487.90 \pm 45 \mathrm{ppm}$, and an estimated planet radius of $2.32 \pm$ $0.46 R_{\oplus}$. The candidate passed all the tests from the Threshold Crossing Event (TCE) Data Validation Report (DVR; Twicken et al. 2018; Li et al. 2019).

We used the publicly available Presearch Data Conditioning (PDC-SAP) flux time series (e.g., Twicken et al. 2010; Smith et al. 2012) provided by SPOC for the transit modeling, which is corrected for common trends and artifacts, for crowding, and for the finite flux fraction in the photometric aperture. Figure 1 shows the 2 min cadence TESS light curve, along with the phasefolded curve for TOI $1062 \mathrm{~b}$.

\subsection{Ground-based photometry with LCOGT}

We obtained ground-based time series follow-up photometry of full transits of TOI 1062.01 on 24 August 2019 in $i^{\prime}$ band and on 22 September 2020 in Pan-STARSS $z$-shortband from the Las Cumbres Observatory Global Telescope (LCOGT; Brown et al. 2013) $1.0 \mathrm{~m}$ network node at the South Africa Astronomical Observatory. We used the TESS Transit Finder, which is a customized version of the Tapir software package (Jensen 2013), to schedule the observations. The $4096 \times 4096$ LCO SINISTRO cameras have an image scale of $0.389^{\prime \prime}$ per pixel, providing a field of view of $26^{\prime} \times 26^{\prime}$. The standard LCOGT BANZAI pipeline was used to calibrate the images, and the photometric data were extracted with the AstroImageJ (AIJ) software package (Collins et al. 2017).

The initial $i^{\prime}$-band observation intentionally saturated the target star to check for a faint nearby eclipsing binary (NEB) that could be contaminating the TESS photometric aperture. To account for possible contamination from the wings of neighboring star PSFs, we searched for NEBs out to 2.5' from the target star. If fully blended in the SPOC aperture, a neighboring star that is fainter than the target star by 8.8 magnitudes 

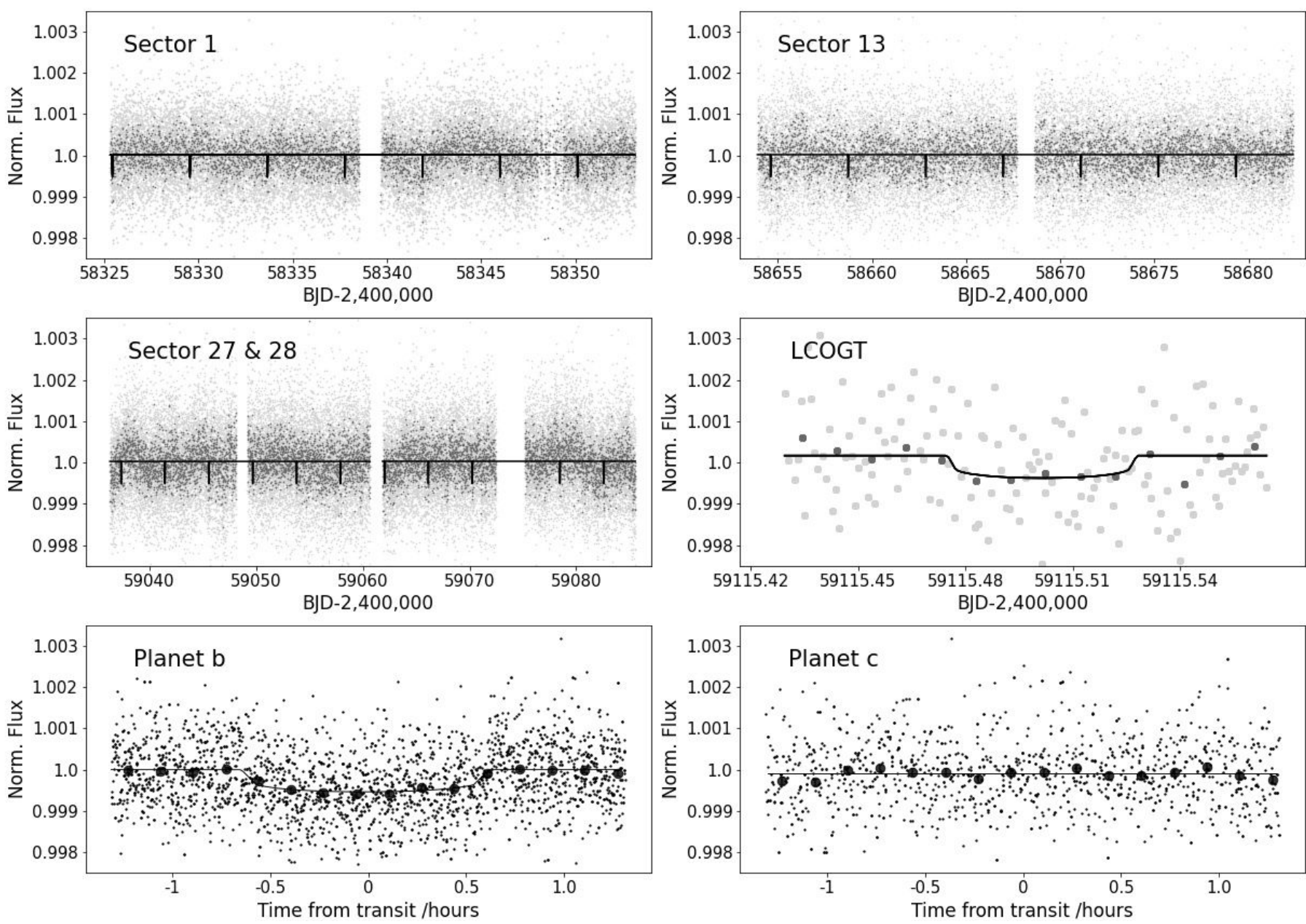

Fig. 1. TESS photometry from the of TOI 1062 from Sectors 1 (upper left), 13 (upper right), and Sectors 27 and 28 (middle left), and LCOGT photometry (middle right). The TESS light curves correspond to the PDC-SAP flux time series provided by SPOC. The TESS full light curve with the 2 min cadence data is shown in light gray, and the same data binned to $10 \mathrm{~min}$ is in dark gray. The gaps in the data coverage are due to observation interruptions of the TESS spacecraft that occur after each TESS orbit of 13.7 days. The dots of the LCOGT are bigger for better visualization. The lower panels shows the phase-folded TESS light curves for TOI 1062b (left) and TOI 1062c (right) with the 2 min cadence data in light gray and binned to $10 \mathrm{~min}$ in black. We find that TOI 1062c does not transit.

in TESS band could produce the SPOC-reported flux deficit at mid-transit (assuming a $100 \%$ eclipse). To account for possible delta-magnitude differences between TESS band and $i^{\prime}$ band, we included an extra 0.5 magnitudes fainter (down to TESSband magnitude 18.3). Our search ruled out NEBs in all 24 neighboring stars that meet our search criteria. The $z$-shortband observation was defocused and exposed appropriately to measure the TOI 1062.01 light curve at high precision. For the data reduction process we used a $10.5^{\prime \prime}$ radius aperture and selected four comparison stars with brightness comparable to that of the target star. The inner and outer radii of the background annulus were then set to the corresponding AIJ calculated values of 18.7" and $28^{\prime \prime}$, respectively. This combination was chosen as it minimized the amount of noise in the light curve. A $0.5 \mathrm{ppt}$ transit, with model residuals of $0.3 \mathrm{ppt}$ in $10 \mathrm{~min}$ bins, was detected in the uncontaminated target aperture.

\subsection{HARPS follow-up}

We collected 90 high-resolution spectra of TOI 1062 using the High Accuracy Radial Velocity Searcher (HARPS) spectrograph mounted at the ESO $3.6 \mathrm{~m}$ telescope of La Silla Observatory, Chile (Mayor et al. 2003), with the goal of precisely determining the mass of the planet candidate and searching for additional planets in the system. The observations were carried out as part of the NCORES large program (ID 1102.C-0249, PI: Armstrong) between 31 August 2019 and 17 January 2020. HARPS is a stabilized high-resolution (R 115000) echelle spectrograph which can reach sub- $\mathrm{m} \mathrm{s}^{-1} \mathrm{RV}$ precision (Mayor et al. 2003). The instrument was used in high-accuracy mode (HAM), with a $1^{\prime \prime}$ fiber on the star and another one to monitor the sky background. We used exposure times of $30 \mathrm{~min}$.

The standard HARPS Data Reduction Software (DRS) was used to reduce the data, using a $\mathrm{K} 0$ mask for both the crosscorrelation function (CCF; Pepe et al. 2002; Baranne et al. 1996) and the color correction, and reaching a typical signalto-noise ratio per pixel of 60 and a photon-noise uncertainty of $1.4 \mathrm{~m} \mathrm{~s}^{-1}$. For each spectrum the usual activity indicators (Sindex, $\mathrm{H} \alpha$-index, Na-index, $\mathrm{Ca}$-index, $\log R_{\mathrm{HK}}^{\prime}$ ), the full width half maximum (FWHM), the line bisector, and the contrast of the CCF were measured.

\subsection{High-resolution imaging}

To check for the presence of contaminating stars in the TESS photometric aperture, TOI 1062 was observed on 14 January 


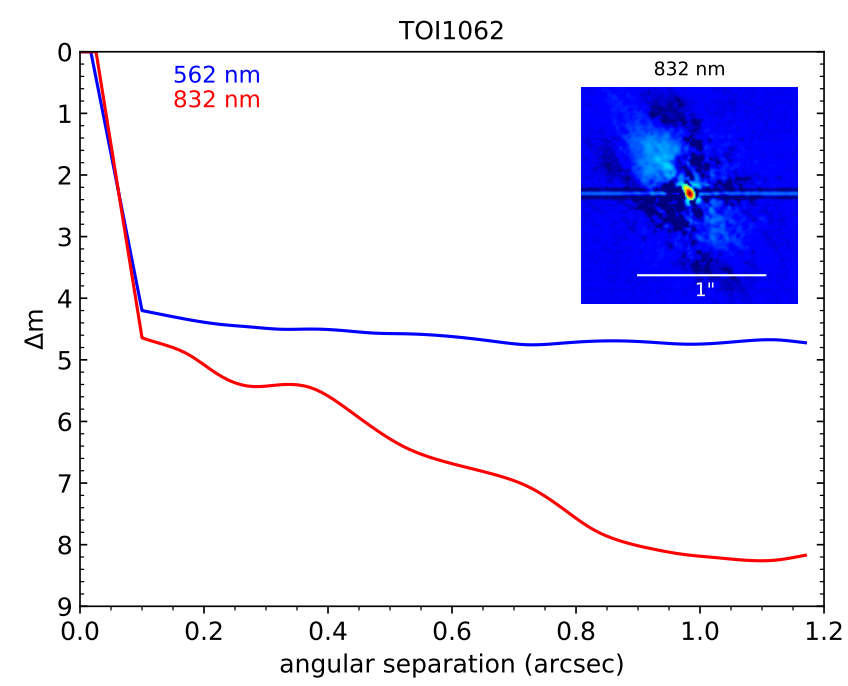

Fig. 2. Gemini/Zorro contrast curves and $1.2^{\prime \prime} \times 1.2^{\prime \prime}$ reconstructed image of the $832 \mathrm{~nm}$ band.

2020 using the Zorro speckle imager (Scott 2019), mounted on the $8.1 \mathrm{~m}$ Gemini South telescope in Cerro Pachon, Chile. Zorro uses high-speed electron-multiplying CCDs (EMCCDs) to simultaneously acquire data in two bands centered at 562 and $832 \mathrm{~nm}$. The data is reduced following the procedures described in Howell et al. (2011), and provides output data including a reconstructed image and robust limits on companion detections. The contrast achieved in the resulting reconstructed image is shown in Fig. 2. We see that the Zorro speckle images reach a contrast of $\Delta$ mag $=8.25$ at a separation of $1.1^{\prime \prime}$ in the $832 \mathrm{~nm}$ band, and cover a spatial range of 9.9-98.6 au around the star with contrasts between 5 and 8 mag in both bands, showing no close companions to TOI $1062 \mathrm{~b}$.

\section{Host star fundamental parameters}

\subsection{Analysis of the spectrum}

The stellar atmospheric parameters $\left(T_{\text {eff }}, \log g\right.$, and $\left.[\mathrm{Fe} / \mathrm{H}]\right)$ and respective error bars were derived using the methodology described in Sousa (2014); Santos et al. (2013). Briefly, we make use of the equivalent widths (EWs) of iron lines, as measured in the combined HARPS spectrum of TOI 1062 using the ARES $\mathrm{v} 2 \operatorname{code}^{1}$ (Sousa et al. 2015), and we assume ionization and excitation equilibrium. This method makes use of a grid of Kurucz model atmospheres (Kurucz 1993) and the radiative transfer code MOOG (Sneden 1973). This approach leads to the following stellar parameters: $T_{\text {eff }}=5328 \pm 56 \mathrm{~K},[\mathrm{Fe} / \mathrm{H}]=0.14 \pm 0.04 \mathrm{dex}$, and $\log g=4.55 \pm 0.09$. This is in agreement with the $\log g$ derived making use of the Gaia parallax and luminosity (Santos et al. 2004) which gives a value of 4.53. Using the Torres et al. (2010) calibration leads to a stellar mass of $0.94 \pm 0.02 M_{\odot}$ and radius of $0.84 \pm 0.09 R_{\odot}$. These values are used as priors in the global fit with juliet in Sect. 4.

\subsection{Analysis of the spectral energy distribution}

As an independent determination of the stellar parameters, we also performed an analysis of the broadband spectral energy

\footnotetext{
1 The most recent version of the ARES code (ARES v2) can be downloaded at http://www. astro. up.pt/ sousasag/ares.
}

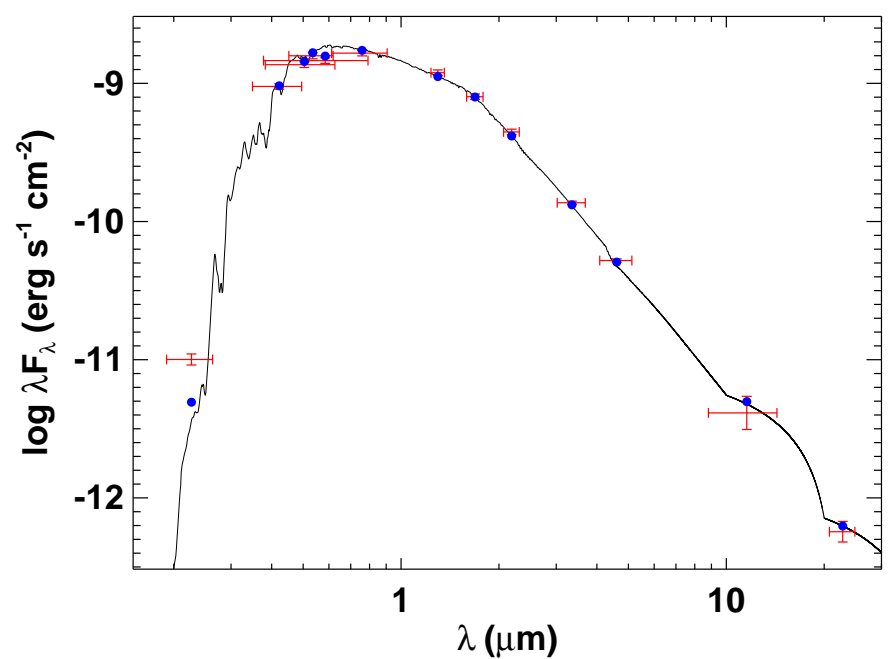

Fig. 3. Spectral energy distribution of TOI 1062. Red symbols represent the observed photometric measurements; the horizontal bars represent the effective width of the passband. Blue symbols are the model fluxes from the best-fit Kurucz atmosphere model (black).

distribution (SED) of the star together with the Gaia DR2 parallax (adjusted by +0.08 mas to account for the systematic offset reported by Stassun \& Torres 2018), in order to determine an empirical measurement of the stellar radius, following the procedures described in Stassun \& Torres (2016), and Stassun et al. (2017, 2018). We pulled the $B_{T} V_{T}$ magnitudes from Tycho-2; the $B V i$ magnitudes from APASS; the $J H K_{S}$ magnitudes from 2MASS; the W1-W4 magnitudes from WISE; the $G, G_{\mathrm{BP}}, G_{\mathrm{RP}}$ magnitudes from Gaia; and the near-ultraviolet (NUV) magnitude from GALEX. Together, the available photometry spans the full stellar SED over the wavelength range 0.2-22 $\mu \mathrm{m}$ (see Fig. 3).

We performed a fit using Kurucz stellar atmosphere models, using the effective temperature $\left(T_{\mathrm{eff}}\right)$, metallicity $([\mathrm{Fe} / \mathrm{H}])$, and surface gravity $(\log g)$ adopted from the spectroscopic analysis. The only additional free parameter is the extinction $\left(A_{\mathrm{V}}\right)$, which we fixed to be zero due to the star's proximity. The resulting fit is very good (Fig. 3) with a reduced $\chi^{2}$ of 1.8. The reduced $\chi^{2}$ is improved to 1.2 by excluding the GALEX NUV flux, which exhibits a modest excess; using the empirical relations of Findeisen et al. (2011), the GALEX NUV excess implies an activity level $\log R_{\mathrm{HK}}^{\prime}=-4.9 \pm 0.1$, consistent with the spectroscopically determined value.

Integrating the (unreddened) model SED gives the bolometric flux at Earth, $F_{\text {bol }}=2.429 \pm 0.057 \times 10^{-9} \mathrm{erg} \mathrm{s}^{-1} \mathrm{~cm}^{-2}$. Taking the $F_{\text {bol }}$ and $T_{\text {eff }}$ together with the Gaia DR2 parallax gives the stellar radius $R_{\star}=0.838 \pm 0.020 R_{\odot}$. In addition, we can use the $R_{\star}$ together with the spectroscopic $\log g$ to obtain an empirical mass estimate of $M_{\star}=0.91 \pm 0.19 M_{\odot}$, which is consistent with that obtained via empirical relations of Torres et al. (2010) and a 6\% error from the empirical relation itself, $M_{\star}=0.97 \pm 0.06 M_{\odot}$. These values are in agreement with those derived from the spectral analysis in Sect. 3.1.

\subsection{Rotational period and age}

The stellar age can be derived from empirical rotation-age relations of Mamajek \& Hillenbrand (2008). The HARPS calibration from the CCF-FWHM and the color index B-V gives $v \sin i=$ 
TESS RAW LC - Sectors $27 \& 28$
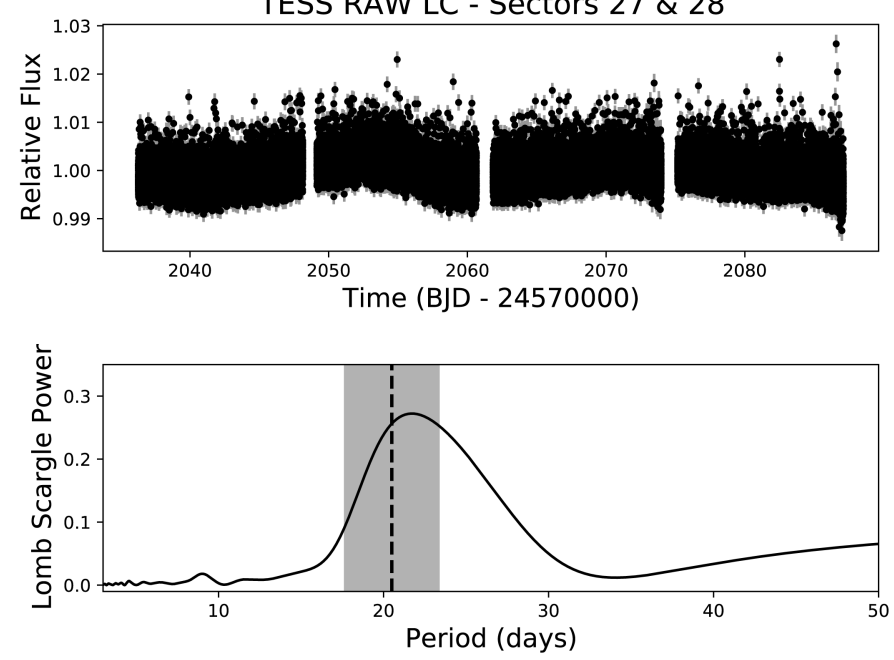

Fig. 4. TESS RAW photometric data of sectors 27 and 28 (top) and the corresponding periodogram. The peak of the periodogram shows a possible rotational modulation with a period of 21.8 days, which is in agreement with the rotational period obtained from HARPS calibration.

$2.13 \pm 0.5 \mathrm{~km} \mathrm{~s}^{-1}$; then together with $R_{\star}$, we estimate the stellar rotation period $P_{\text {rot }} / \sin i=20.5 \pm 2.9$ d. In addition, Fig. 4 shows that the peak of the periodogram of the TESS light curves of Sectors 27 and 28 is at 21.82.4 days. The measured rotational modulation is nearly identical in the other TESS sectors. Another estimate of the expected rotation can be inferred from the average value of the $\mathrm{Ca}$ II $\mathrm{H} \& \mathrm{~K}$ chromospheric activity indicator for TOI 1062, which is $\log R_{\mathrm{HK}}^{\prime}=-4.80 \pm 0.05$. Using the empirical relations in Mamajek \& Hillenbrand (2008) we get a rotation period of $21.9^{+3}$ days, in agreement with the result derived from the HARPS calibration and the modulation of the RAW light curves. Another way of constraining the rotational period of the star consists of fitting the RVs with time-dependent Gaussian processes (GPs). We use the celerite package (e.g., ForemanMackey et al. 2017) with a quasi-periodic kernel, and obtain a rotational period of $22.4_{-6.7}^{+4.6}$ days, which is in agreement with the previous estimations.

Using the rotational period obtained from the TESS data and the relations of Mamajek \& Hillenbrand (2008), the age estimate is $\tau_{\star}=2.5 \pm 0.3 \mathrm{Gyr}$. Because the $v \sin i$ is a projected rotational velocity, we note that it is a lower limit on the true rotational velocity, so $P_{\text {rot }} / \sin i$ is an upper limit on the true $P_{\text {rot }}$, which implies that the age estimate above provides an upper limit to the stellar age.

We also attempted to derive the stellar age by making use of chemical clocks (e.g., Delgado Mena et al. 2019); however, we obtain a value much higher than with other methods described below. The reason is that the low $T_{\text {eff }}$ of TOI 1062 lies at the limit of applicability of such empirical relations, and thus we did not use the age provided by this method.

\subsection{Stellar abundances}

Stellar abundances of refractory elements were derived using the classical curve-of-growth analysis method assuming local thermodynamic equilibrium (e.g., Adibekyan et al. 2012; Delgado Mena et al. 2017). Abundances of the volatile elements $C$ and $\mathrm{O}$ were derived following the method of Delgado Mena et al. (2010); Bertran de Lis et al. (2015). Since the two spectral lines of oxygen are usually weak and the $6300.3 \AA$ line is blended with
$\mathrm{Ni}$ and $\mathrm{CN}$ lines, the EWs of these lines were manually measured with the task splot in IRAF. All the $[\mathrm{X} / \mathrm{H}]$ ratios are obtained by doing a differential analysis with respect to a high $\mathrm{S} / \mathrm{N}$ solar (Vesta) spectrum from HARPS. The obtained values for this star are normal considering its metallicity except for the lower-than-expected value of oxygen. We find a high dispersion between both oxygen indicators that has to be taken with caution due to the difficulty of measuring reliable oxygen abundances for cool metal-rich stars. The stellar abundances of the elements are presented in Table 1.

\subsection{Signal identification}

In order to search for periodicities in the RV data we computed the $l_{1}$ periodogram (e.g., Hara et al. 2017, 2020). This tool is designed to find periodicities in time series data corresponding to exoplanets detected using radial velocity data. It can be used similarly to a typical Lomb-Scargle periodogram or its variants (e.g., Baluev 2008), but it is based on a different principle. The $l_{1}$ periodogram can analyze the radial velocity without the need to estimate the frequency iteratively, using the theory of compressed sensing adapted for handling correlated noise. Instead of fitting a sinusoidal function at each frequency as the LombScargle-type periodograms, the $l_{1}$ periodogram searches directly for a representation of the input signal as a sum of sinusoids. As a result, all the frequencies of the signal are searched simultaneously. This relies on the so-called sparse recovery tools, which are designed to find a representation of an input signal as a linear combination of sine functions.

The $l_{1}$ periodogram outputs a figure that has a similar aspect to that of a standard Lomb-Scargle-type periodogram, but with fewer peaks due to aliasing. We use the $l_{1}$ periodogram of the HARPS data on a grid of frequencies between 0.01 and 0.3 cycles per day. In order to measure the significance of a possible signal the false alarm probability (FAP) is calculated, which encodes the probability of measuring a peak of a given height conditioned on the assumption that the data consists of Gaussian noise with no periodic component. Typically, a signal at the $1 \%$ FAP level is considered suggestive, and a $0.1 \%$ FAP level statistically significant. Figure 5 shows two $l_{1}$ periodograms of the HARPS RVs. The top panel corresponds to the undetrended RVs. In this case we detect three significant signals at $4.11 \mathrm{~d}$ (FAP $10^{-4} \%$ ), 7.98 days (FAP $7.10^{-6} \%$ ), and 26.6 days (FAP $\left.3.10^{-5} \%\right)$. Although the coefficient amplitude of the second and third peaks are relatively close, their FAP values can differ significantly since it is estimated in a sequential manner: the highest peak is computed against zero, the second peak against the first, and the third peak against the second. Figure 6 clearly shows the effects of activity in the FWHM at the end of the time series. It suggests that the star was more active at that moment, probably due to one spot crossing event during one single rotational period, standing for only 20 days (from BJD 58 830-58 850). However, we find that the signal at 26.6 days disappears when we detrend the RVs using GP modeling with SPLEAF (e.g., Delisle et al. 2020) (see bottom panel of Fig. 5). The GP is trained on the $\log R_{\mathrm{HK}}^{\prime}$ indicator, and then it is used to detrend the RVs using a linear fit. This method gives two significant peaks at $4.11 \mathrm{~d}$ with FAP $6.10^{-5} \%$ and 8.00 days with FAP $4.10^{-4} \%$.

Another way to detrend the stellar activity is to fit the correlations between activity indicators as the full width at half maximum (FWHM), the bisector or the S-index, and subtract them in order to correct for any long-term effects resulting from a magnetic cycle. We do so dividing the data into different time regimes selected in order to maximize the correlation of the RV 

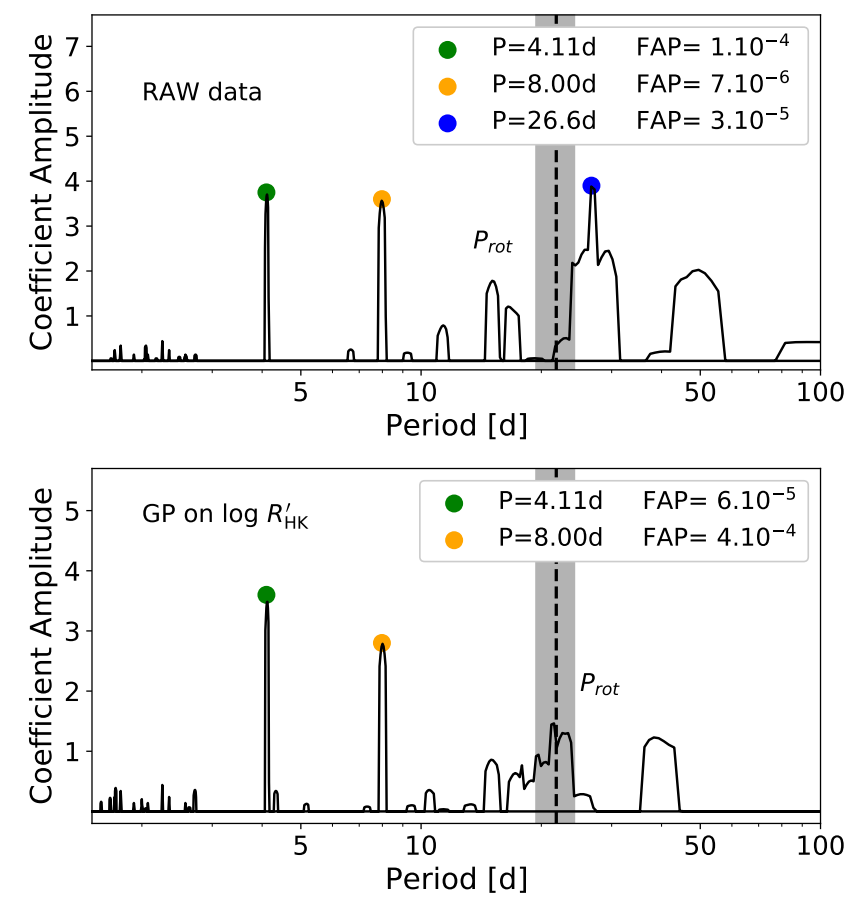

Fig. 5. Undetrended RVs (top) and detrended RVs (bottom) obtained via the $l_{1}$ periodogram using Gaussian processes on the $\log R_{\mathrm{HK}}^{\prime}$ indicator with SPLEAF. The periods at which the significant peaks occur $\left(\right.$ FAP $<10^{-3}$ ) are color-coded (see inset). The stellar rotational period determined through the TESS data is highlighted in gray.

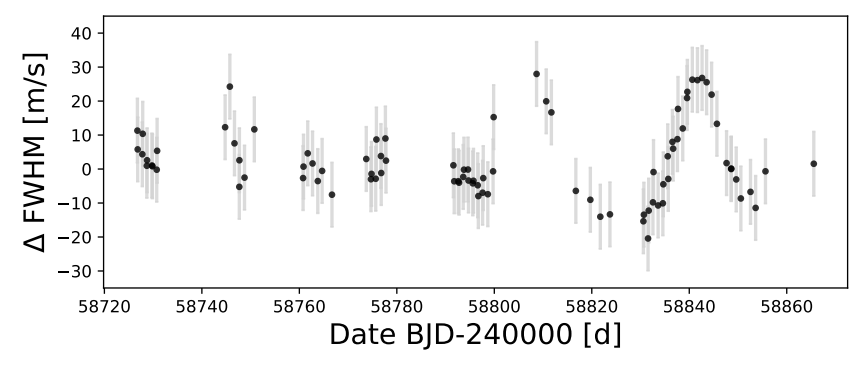

Fig. 6. Time series of the CCF-FWHM.

data with the activity indicators in each bin. This approach also identifies two peaks at $4.11 \mathrm{~d}$ with FAP $7.10^{-8} \%$ and 8.01 days with FAP $3.10^{-4} \%$. Finally, we also find the same two significant signals when we do not use the data TBJD 58730 -58750, which corresponds to periods when the star was more active. The signal at 26.6 days only appears when using the full undetrended RV sample: it is clearly affected by stellar activity and it is within $1.6 \sigma$ of the rotation period derived from the chromospheric activity indicator. We therefore conclude that only the peaks at $\sim 4.11$ days and $\sim 8$ days are caused by planets. In addition, even though the $\sim 8$-day signal is nearly double the $\sim 4$.11-day signal, in all the cases presented we find the 8-day signal after removing the $~ 4.11$-day signal. We also evaluate the evidence of the second planet by comparing the difference in the Bayesian information criterion $(\triangle B I C)$ between the one-eccentric-planet and the twoeccentric-planets models. We obtain a value of $\triangle \mathrm{BIC}=11$, which suggests strong evidence.

\subsection{Global fit with juliet}

We determine the planetary parameters using the publicly available software juliet (e.g., Espinoza et al. 2019). juliet allows
Table 2. Prior parameter distribution of the global fit with juliet.

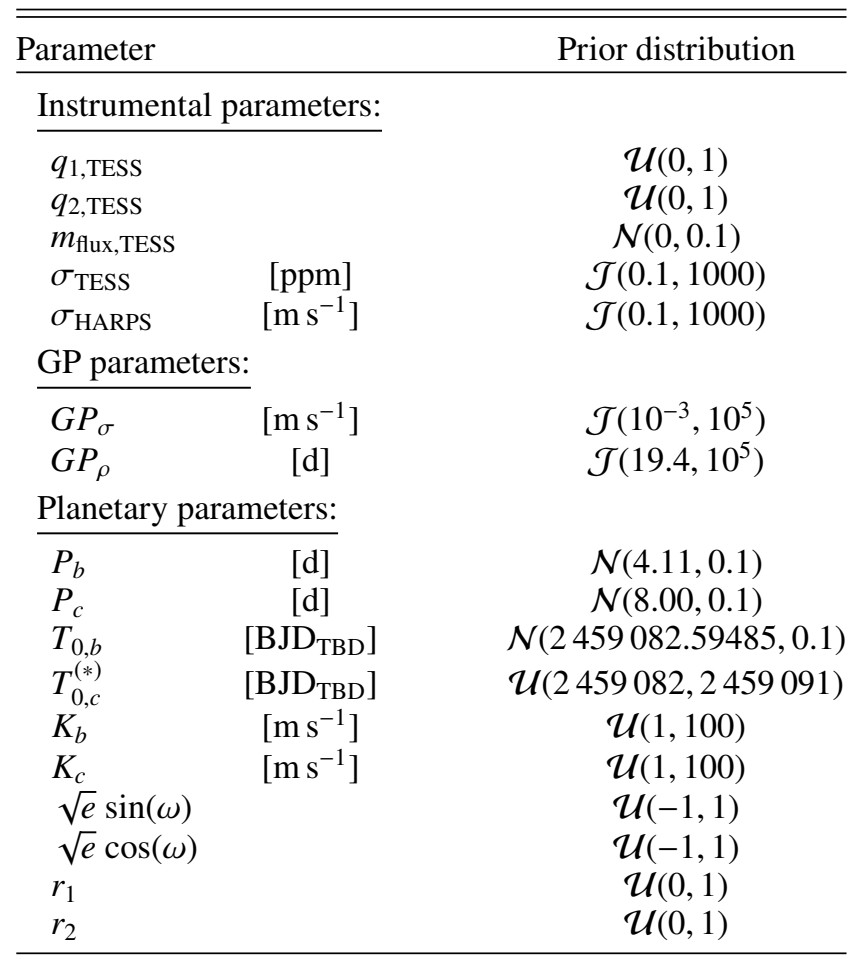

Notes. ${ }^{(*)} \mathcal{U}(a, b)$ indicates a uniform distribution between $a$ and $b$; $\mathcal{J}(a, b)$ a Jeffrey or log-uniform distribution between $a$ and $b$; and $\mathcal{N}(a, b)$ a normal distribution with mean $a$ and standard deviation $b$.

us to jointly fit the TESS and LCOGT photometry and the HARPS radial velocities with GP. The software is built on several publicly available tools used to model the photometric data (the batman package; Kreidberg 2015), the radial velocities (the radvel package; Fulton et al. 2018), and also to incorporate GP (the celerite package, Foreman-Mackey et al. 2017). The parameter space is explored using nested sampling, with the MultiNest algorithm (e.g., Feroz \& Hobson 2008) in its Python implementation, PyMultiNest (e.g., Buchner et al. 2014). In addition, juliet computes the Bayesian evidence using dynesty (e.g., Speagle 2020), a Python package that estimates Bayesian posteriors and evidence using dynamic nested sampling. In short, nested sampling algorithms work as follows. The algorithm samples a number of live points randomly from the prior distribution, and the likelihood is evaluated at each of these points. At each iteration the point with the lowest likelihood is replaced by a new sampled point, keeping the number of live points constant. This process is continued until Bayesian evidence reaches a specified value. The number of live points used has to be large enough to adequately sample the parameter space.

The transit model fits the stellar density $\rho_{\star}$ together with the planetary and jitter parameters. For the stellar density we use the value obtained in Sect. 3, and the priors of the orbital parameters of the inner planet are taken from ExoFOP. We use the quadratic limb darkening coefficients $\left(q_{1}, q_{2}\right)$ introduced by Kipping (2013) for the photometric data, since it was shown to be appropriate for space-based missions (Espinoza \& Jordán 2015). In addition, instead of fitting the planet-to-star radius ratio and the impact parameter of the orbit, we use the parameterization introduced in Espinoza (2018) and fit the parameters $r_{1}$ and $r_{2}$ to make sure the full exploration of physically plausible values in the $(\mathrm{p}, \mathrm{b})$ space. We parameterize the eccentricity and 

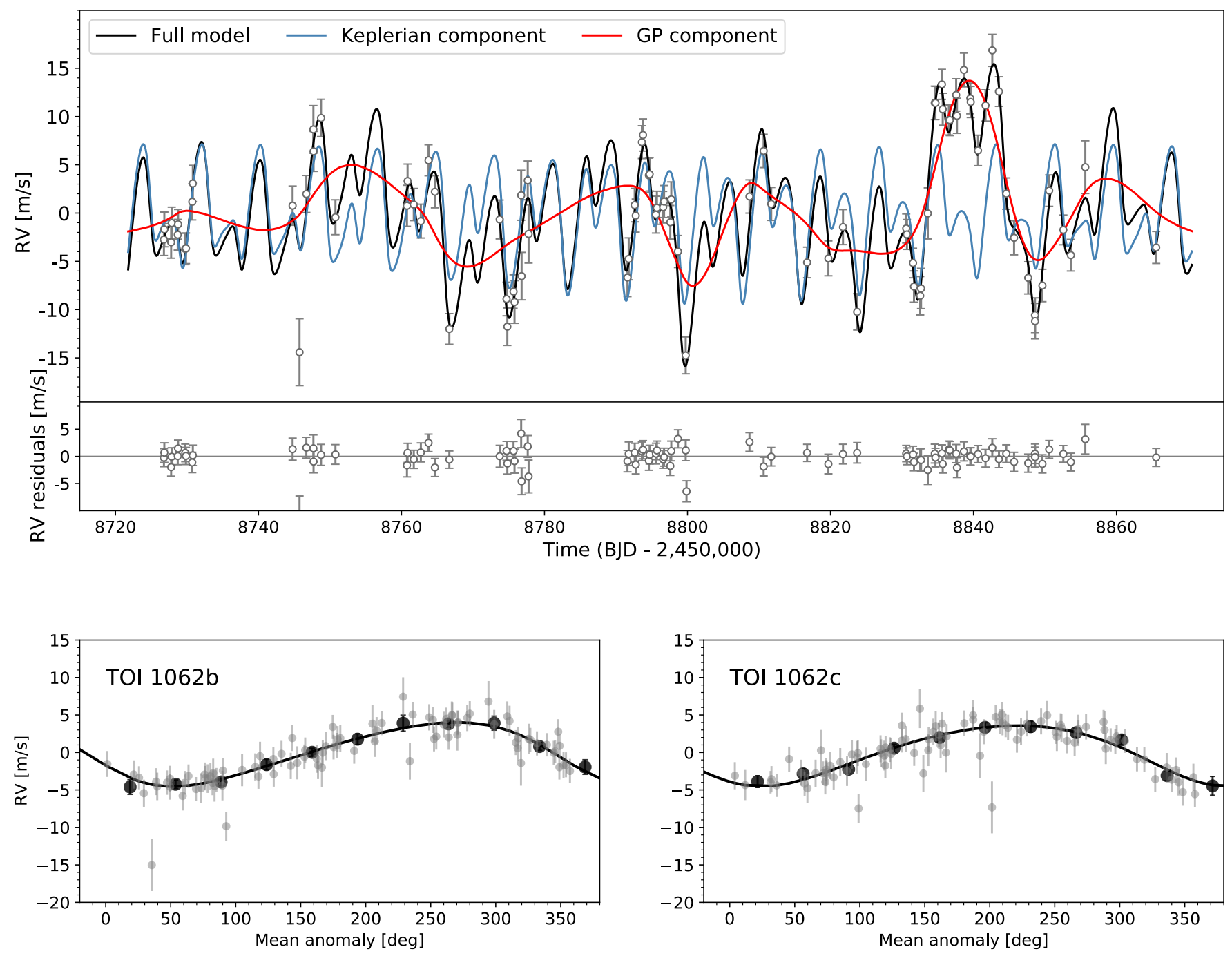

Fig. 7. HARPS RVs for TOI 1062 with a two planet model including eccentric orbits and Gaussian processes with Matern kernel. Top panel: RVs along with the residuals to the best fit right below the time series. Bottom panel: phase-folded data for each planet. The gray dots represent the observed RVs and the black dots the binned data.

the argument of periastron with $\sqrt{e} \sin \omega$ and $\sqrt{e} \cos \omega$, always ensuring that $e \leq 1$. We use the celerite aproximate Matern multiplied by exponential kernel to account for the stellar activity. The fit obtained with this kernel is nearly identical to the one obtained with other kernels as the quasi-periodic or the exp-sine-squared kernel.

We fit both planets with juliet and find no hint of transit for TOI 1062c, as shown in Fig. 1. The priors used are shown in Table 2, the final median planetary parameters determined by the juliet fit are listed in Table 3, and the RV fit is presented in Fig. 7. We find that TOI 1062b has an orbital period of 4.114 days, a radius of $2.265_{-0.091}^{+0.096} R_{\oplus}$, a mass of $10.15_{-0.84}^{+0.81}$ $M_{\oplus}$, and a density of $4.85_{-0.74}^{+0.84} \mathrm{~g} \mathrm{~cm}^{-3}$, slightly below the Earth's mean density. Nevertheless, as discussed in detail in Sect. 5, its composition and internal structure are unlikely to be similar to that of the Earth. We find an eccentricity of $0.18_{-0.06}^{+0.07}$ for TOI-1062b and $0.14 \pm 0.07$ for TOI-1062c. Figure 8 shows the eccentricity against the orbital period for the population of observed exoplanets from the NASA Exoplanet Archive, and we see that the TOI-1062b value is slightly higher than usual for its orbital period. However, it may not be significant due to the bias towards higher eccentricities for nearly circular planets

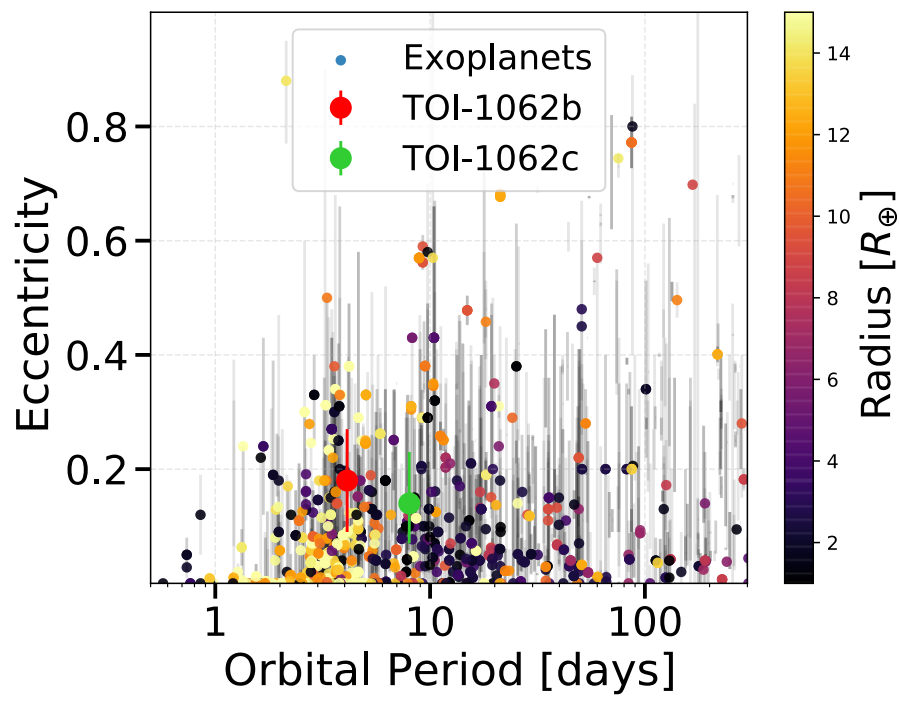

Fig. 8. Orbital eccentricity against orbital period for observed exoplanets from the NASA Exoplanet Archive. The color represents the planetary radius (see color scale at right). 
Table 3. TOI-1062 parameters from juliet: median and $68 \%$ confidence interval.

\begin{tabular}{|c|c|c|c|}
\hline \multicolumn{4}{|c|}{ Instrumental parameters: } \\
\hline$q_{1, \mathrm{TESS}}$ & Quadratic limb-darkening parametrization & \multicolumn{2}{|l|}{$0.845_{-0.033}^{+0.035}$} \\
\hline$q_{2, \mathrm{TESS}}$ & Quadratic limb-darkening parametrization & \multicolumn{2}{|l|}{$0.113_{-0.067}^{+0.114}$} \\
\hline$m_{\text {flux,TESS }}$ & Offset relative flux & \multicolumn{2}{|l|}{$-0.0000217_{-0.0000017}^{+0.00 /}$} \\
\hline$\sigma_{\text {TESS }}$ & Jitter (ppm) & \multicolumn{2}{|l|}{$118.42_{-5.03}^{+5.27}$} \\
\hline$\sigma_{\text {HARPS }}$ & Jitter $\left(\mathrm{m} \mathrm{s}^{-1}\right)$ & \multicolumn{2}{|l|}{$1.141_{-0.101}^{+0.057}$} \\
\hline \multicolumn{4}{|c|}{ Parameters of the GP with quasi-periodic kernel: } \\
\hline$G P_{\sigma}$ & Amplitude $\left(\mathrm{m} \mathrm{s}^{-1}\right)$ & $21.19_{-2.6}^{+3.1}$ & \\
\hline$G P_{\rho}$ & Time-scale (days) & $21.80_{-1.33}^{+1.91}$ & \\
\hline \multicolumn{2}{|c|}{ Planetary parameters: } & $\mathrm{b}$ & $\mathrm{c}$ \\
\hline$P$ & 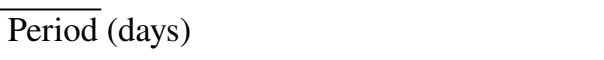 & $4.11296_{-0.0015}^{+0.0015}$ & $7.972_{-0.024}^{+0.018}$ \\
\hline$T_{0}$ & Time of transit center* $\left(\mathrm{BJD}_{\mathrm{TBD}}\right)$ & $2459082.59_{-0.09}^{+0.09}$ & $2459087.61_{-1.63}^{+1.84}$ \\
\hline$K$ & Radial velocity semi-amplitude $\left(\mathrm{m} \mathrm{s}^{-1}\right)$ & $4.27_{-0.33}^{+0.31}$ & $4.05_{-0.41}^{+0.46^{-1.05}}$ \\
\hline$\sqrt{e} \sin (\omega)$ & Parametrization for $e$ and $\omega$ & $0.14_{-0.08}^{+0.08}$ & $-0.02_{-0.06}^{+0.41}$ \\
\hline$\sqrt{e} \cos (\omega)$ & Parametrization for $e$ and $\omega$ & $-0.07_{-0.06}^{+0.05}$ & $-0.09_{-0.81}^{+0.12}$ \\
\hline$r_{1}$ & Parametrization for $p$ and $b$ & $0.908_{-0.007}^{+0.006}$ & - \\
\hline$r_{2}$ & Parametrization for $p$ and $b$ & $0.0235_{-0.0004}^{+0.0003}$ & - \\
\hline \multicolumn{2}{|c|}{ Derived transit and RV parameters: } & $\mathrm{b}$ & $\mathrm{c}$ \\
\hline $\bar{e}$ & Eccentricity or the orbit & $0.177_{-0.064}^{+0.067}$ & $0.140_{-0.069}^{+0.073}$ \\
\hline$\omega$ & Argument of periastron (deg) & $117_{-19}^{+27}$ & $159_{-35}^{+16}$ \\
\hline$i$ & Inclination (deg) & $85.913_{-0.041}^{+0.062}$ & - \\
\hline$p=R_{p} / R_{\star}$ & Planet-to-star radius ratio & $0.0234_{-0.0004}^{+0.0004}$ & - \\
\hline$b$ & Impact parameter of the orbit & $0.861_{-0.009}^{+0.007}$ & - \\
\hline \multicolumn{2}{|c|}{ Derived physical parameters: } & $\mathrm{b}$ & $\mathrm{c}$ \\
\hline$M_{p}$ & Planetary mass $\left(M_{\oplus}\right)$ & $10.15_{-0.84}^{+0.81}$ & - \\
\hline$R_{p}$ & Planetary radius $\left(R_{\oplus}\right)$ & $2.265_{-0.091}^{+0.096}$ & - \\
\hline$\rho_{p}$ & Planetary density $\left(\mathrm{g} \mathrm{cm}^{-3}\right)$ & $4.85_{-0.74}^{-0.091}$ & - \\
\hline$a_{p}$ & Semi-mayor axis (AU) & $0.052_{-0.025}^{+0.024}$ & $0.080_{-0.012}^{+0.013}$ \\
\hline$S$ & Insolation $\left(S_{\oplus}\right)$ & $232_{-10}^{+11}$ & $95_{-4}^{+5}$ \\
\hline$T_{\mathrm{q}}$ & Equilibrium temperature $(K)$ & $1077_{-9}^{+10}$ & $859_{-8}^{+9}$ \\
\hline$M_{P} \sin i$ & Minimum planetary mass $\left(M_{\oplus}\right)$ & $10.15_{-0.85}^{+0.82}$ & $9.78_{-1.18}^{+1.26}$ \\
\hline
\end{tabular}

Notes. For TOI 1062c $T_{0}$ corresponds to the time when the planet would have transited.

(Lucy \& Sweeney 1971). TOI 1062 c instead is found to have an orbital period of $7.988 \pm 0.04$ days and a minimum mass of $9.78_{-1.18}^{+1.26} M_{\oplus}$. TOI $1062 \mathrm{c}$ is in nearly $2: 1$ motion resonance with its inner companion.

\section{Discussion}

\subsection{Internal structure}

In order to characterize the internal structure of TOI $1062 \mathrm{~b}$, we modeled its interior considering a pure-iron core, a silicate mantle, a pure-water layer, and a $\mathrm{H}-\mathrm{He}$ atmosphere. The equations of state (EOSs) used for the iron core are from Hakim et al. (2018), the EOS of the silicate-mantle was calculated with PERPLE_X from Connolly (2009) using the thermodynamic data of Stixrude \& Lithgow-Bertelloni (2011) and assuming Earth-like abundances, and the EOS for the H-He envelope are from Chabrier et al. (2019) assuming a proto-solar composition. For the pure-water layer we used the AQUA EOS from Haldemann et al. (2020). We assumed an envelope luminosity of $L=10^{22.52} \mathrm{erg} \mathrm{s}^{-1}$ (equal to Neptune's luminosity). The thickness values of the planetary layers were set by defining their masses and solving the structure equations. To obtain the transit radius, we followed Guillot (2010) and evaluated the location where the chord optical depth $\tau_{\text {ch }}$ is $2 / 3$. We did not use stellar abundances as an additional constraint since it is not clear whether they are a good proxy for the planetary bulk abundances (Wang et al. 2019; Plotnykov \& Valencia 2020), and it has been shown that the stellar abundances are not always useful for constraining the internal composition (Otegi et al. 2020b).

Figure 9 shows M-R curves tracing the compositions of Earth-like planets (with a CMF $=0.33$ ) and pure water subjected to a stellar radiation of $F / F_{\oplus}=230$ (similar to that of TOI 1062 b). For reference, we also show exoplanets with 

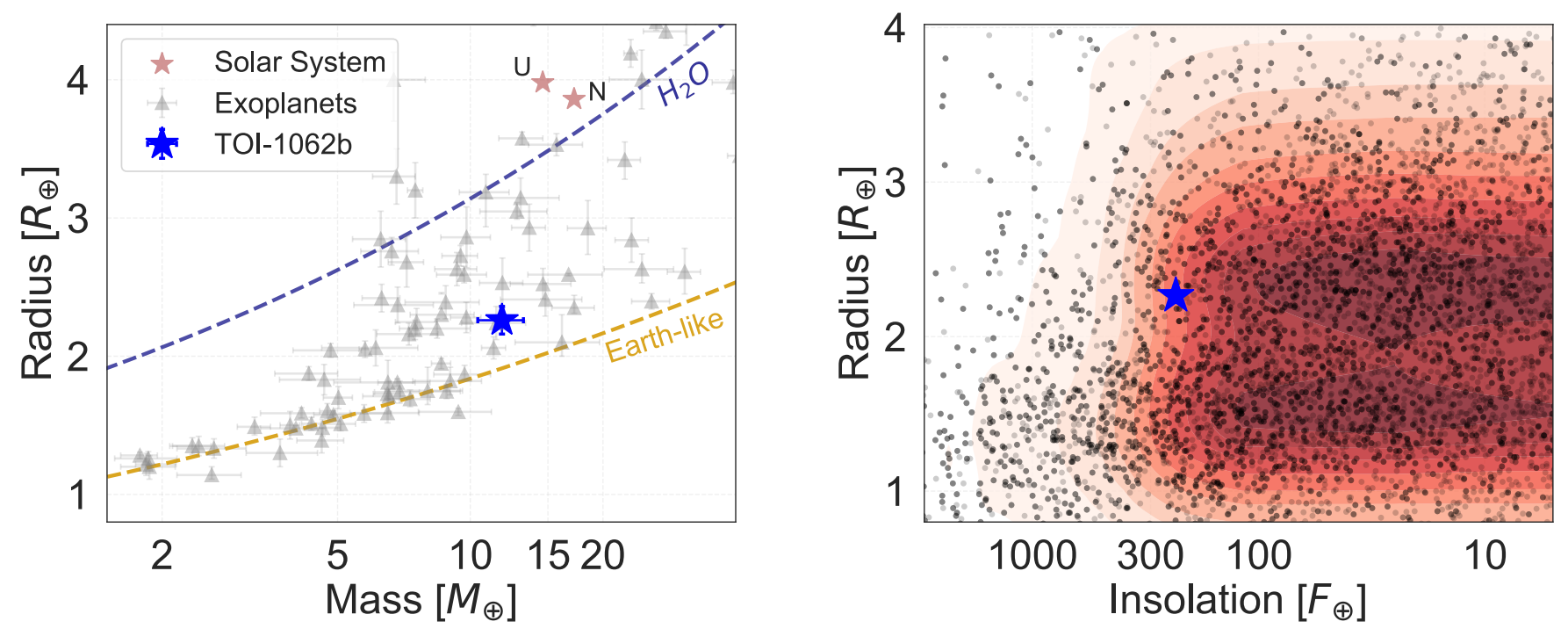

Fig. 9. Mass-radius and insolation-radius diagrams of observed exoplanets. Left: mass-radius diagram of exoplanets with accurate mass and radius determination from Otegi et al. (2020a). Also shown are the composition lines for Earth-like planets and pure water subjected to a stellar radiation of $F / F_{\oplus}=230$ (similar to that of TOI $1062 \mathrm{~b}$ ). Right: radius against insolation flux for known exoplanets from the NASA Exoplanet Archive. The colormap indicates the point density. TOI $1062 \mathrm{~b}$ is represented by a blue star.

accurate and reliable mass and radius determinations (Otegi et al. 2020a, accessible on the Data Analysis Center for Exoplanets, DACE ${ }^{2}$ ). TOI 1062 b sits above the Earth-like curve and below the pure-water curve, suggesting that it contains a small amount of volatile materials of less than $1 \%$ in mass. Figure 9 also displays the insolation flux relative to Earth against radii for the known exoplanets extracted from the NASA Exoplanet Archive, which shows the separate populations of super-Earths and mini-Neptunes. We see that TOI $1062 \mathrm{~b}$ sits in the miniNeptune regime, close to the radius valley. In Otegi et al. (2020a) we identified two distinct populations, volatile-rich and "rocky" exoplanets (those expected to have small amounts of volatiles) separated by the water line. Using these results, we see that TOI $1062 \mathrm{~b}$ lies in the rocky exoplanet regime defined in Otegi et al. (2020a), even if its radius is above the radius valley, suggesting that it is mostly composed of refractory materials by mass.

We used a generalized Bayesian inference analysis using a nested sampling scheme (Buchner et al. 2014) to quantify the degeneracy between various interior parameters and produce posterior probability distributions. Figure 10 shows ternary diagrams of the inferred composition of TOI $1062 \mathrm{~b}$. The ternary diagram shows the degeneracy associated with the determination of the composition of exoplanets with measured mass and radius. We find a median $\mathrm{H}-\mathrm{He}$ mass fraction of $0.1 \%$, which corresponds to a lower bound since enriched $\mathrm{H}-\mathrm{He}$ atmospheres are more compressed, and therefore increase the planetary $\mathrm{H}$ He mass fraction. Formation models suggest that sub-Neptunes are likely formed by envelope enrichment (Venturini \& Helled 2017). We also find that TOI $1062 \mathrm{~b}$ is expected to have a very significant iron core and silicate mantle, accounting for nearly $40 \%$ of the planetary mass and thicknesses of $1 R_{\oplus}$ and $0.5 R_{\oplus}$, respectively. The water layer has an estimated relative mass fraction of $15 \%$. Nevertheless, the degeneracy between the core, silicate mantle, and water layer in this M-R regime is particularly high (Otegi et al. 2020b), and it does not allow accurate estimates of the masses of these constituents. Interior models

2 https://dace.unige.ch/exoplanets/
Table 4. Inferred interior structure properties of TOI 1062b.

\begin{tabular}{lcc}
\hline \hline Constituent & 4-layer [\%] & Without $\mathrm{H}_{2} \mathrm{O}[\%]$ \\
\hline$M_{\text {core }} / M_{\text {total }}$ & $44_{-24}^{+26}$ & $26_{-11}^{+26}$ \\
$M_{\text {mantle }} / M_{\text {total }}$ & $40_{-16}^{+27}$ & $73_{-14}^{+11}$ \\
$M_{\text {water }} / M_{\text {total }}$ & $15_{-6}^{+9}$ & - \\
$M_{\mathrm{H}-\mathrm{He}} / M_{\text {total }}$ & $0.11_{-0.04}^{+0.06}$ & $0.35_{-0.08}^{+0.12}$ \\
\hline
\end{tabular}

cannot distinguish between water and $\mathrm{H}-\mathrm{He}$ as the source of lowdensity material, so we also ran a three-layer model without the $\mathrm{H}_{2} \mathrm{O}$ envelope. Table 4 lists the inferred mass fractions of the core, mantle, water layer, and $\mathrm{H}-\mathrm{He}$ envelope for the four-layer model and for the water-free model. In this case we find that the planet is $0.35 \% \mathrm{H}-\mathrm{He}, 26 \%$ iron, and $73 \%$ rock by mass, setting maximum limits for the atmospheric and rock mass since any water added would decrease these mass fractions.

\subsection{Dynamical analysis}

As a general note for this section, the studies presented here were done under the hypothesis of a co-planar planetary system (i.e., the orbits of planets $b$ and $c$ evolve in the same plane). This is consistent with the observations, since TOI 1062c would not transit if it had the same orbital inclination as TOI 1062b.

\subsubsection{Resonance}

As can be seen from Table 3, the period ratio of the planets is $P_{c} / P_{b}=1.941$. The planet pair thus lies close to the $2: 1$ meanmotion resonance (MMR). How close is the system to the MMR? We explore the structure of the parameter space of TOI-1062 near that resonance in order to investigate its dynamical state.

We therefore designed a two-dimensional section of the parameter space defined by the period ratio on one axis and the eccentricity of the outer planet $e_{c}$ on the other. Our section has a 

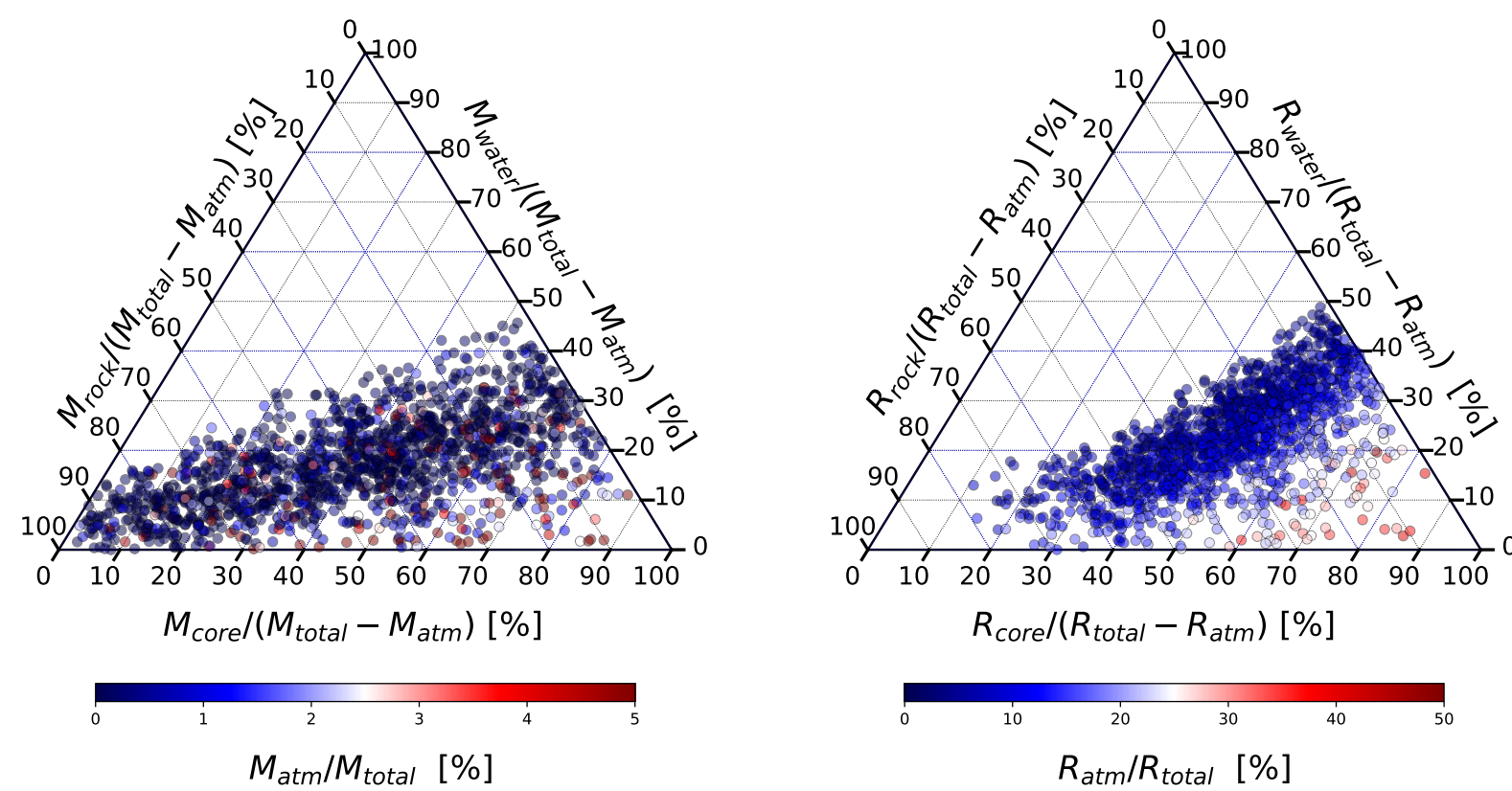

Fig. 10. Ternary diagram of the inferred internal composition of TOI $1062 \mathrm{~b}$. We show the parameter space covered by the posterior distributions in the four-layer model in mass (left) and radius (right).

resolution of $101 \times 101$, meaning that we explore the dynamics of 10201 initial configurations defined by a unique set of $\left(P_{c} / P_{b}, e_{c}\right)$. All the other orbital parameters were initially fixed at the best values reported in Table 3 . We numerically computed the future evolution of each configuration over $20 \mathrm{kyr}$. This was performed with the adaptive time-step high-order N-body integrator IAS15, which is available from the python package REBOUND (Rein \& Liu 2012; Rein \& Spiegel 2015). The perturbative effect of general relativity described in Anderson et al. (1975) was included via the library REBOUNDx (Tamayo et al. 2019). From these numerical simulations, the level of chaos of each configuration was evaluated with the Numerical Analysis of Fundamental Frequencies (NAFF) fast chaos indicator (Laskar et al. 1992; Laskar 1993). The result is presented in Fig. 11.

The NAFF computes precisely, for each planet, the average mean motion $n=\frac{2 \pi}{P}$ over the two halves of the integration and compares these two estimations. Due to the secular constancy of the semi-major axis of regular orbits, this difference should be small in non-chaotic orbits. The higher the drift in the average mean-motion, the more chaotic the orbit. In this work we took as the NAFF of the system the maximum value of this drift over the planetary orbits in logarithmic scale, NAFF $=\max _{i}\left[\log _{10} \frac{\Delta n_{i}}{n_{0, i}}\right]$, where the subscript $i$ refers to the planet $b$ or $c, \Delta n_{i}$ is the drift of the average mean motion over the two halves of the integration, and $n_{0, i}$ is the initial mean motion of planet $i$. The color-coding in Fig. 11 depicts the NAFF defined above: the bluer colors indicate more regular systems. We also distinguish the strongly chaotic configurations from the ones that did not finish the integration because of either a close-encounter or an escape of a body (white boxes). We finally explain our choice of $20 \mathrm{kyr}$ for the total integration time. Over this time span, the planetary orbits in the TOI-1062 system are expected to cover several secular cycles during which the semi-major axes oscillate. Covering several of these cycles allows to properly average the secular variations and isolate the chaotic diffusion. We verified numerically that several secular cycles are made up over an integration.

In this map, the 2:1 mean motion resonance appears clearly as the orange band in the middle of the plot. For the current

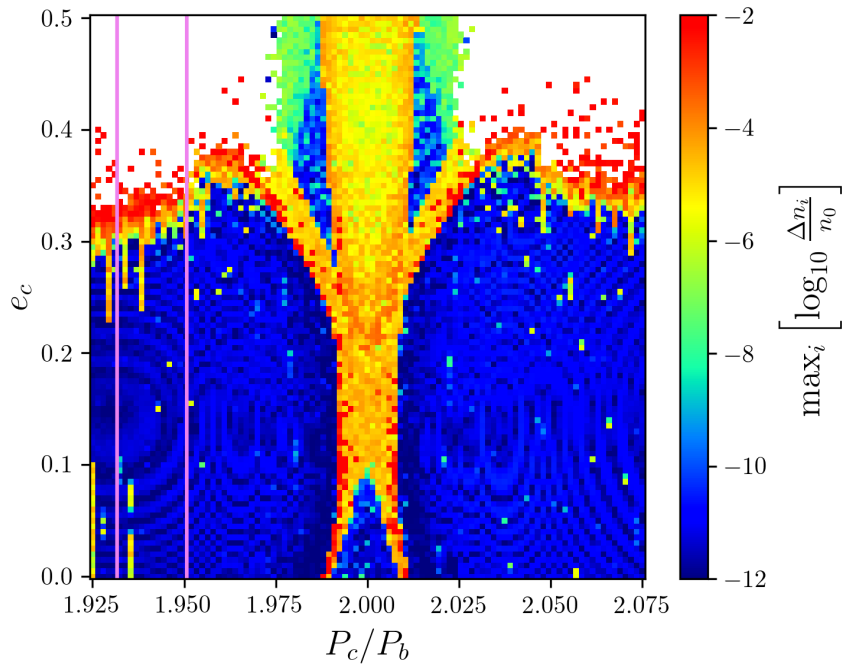

Fig. 11. Chaoticity map around the solution for the two-planet model (Table 3). The eccentricity of the outer planet $e_{c}$ and the period ratio $P_{c} / P_{b}$ are explored on a grid of $101 \times 101$ different configurations. A color is assigned to each box after a short numerical integration, according to its level of chaos using the NAFF indicator (see text).

system's parameters and the resolution of our chaoticity map, this resonance therefore seems chaotic. It is important to note that this picture is highly dependent on the system's parameters. For instance, in this case the arguments of periastron $\omega_{b}$ and $\omega_{c}$ are close to the alignment. The opposite configuration where the orbits are anti-aligned would show a drastically different picture, with a different strength and apparent stability of the 2:1 MMR.

The two vertical lines depict the $1 \sigma$ window of $P_{c}$. With the currently estimated orbital parameters and planet masses, the TOI 1062 system certainly lies outside of the 2:1 MMR. Despite the influence that a revision of the parameters may have on the resonance, it seems very unlikely that this conclusion will change. 
Finally, we note that the uncertainty on the eccentricity of the inner planet $e_{b}$ is quite large. Modifying this parameter will directly impact the strength of the $2: 1 \mathrm{MMR}$ as the resonance width is expected to increase with the eccentricity. The same also applies for the planetary masses.

\subsubsection{Stability constraints}

While the chaoticity map presented above (Fig. 11) is informative about the maximum eccentricity of the outer planet allowed by orbital stability, this estimate should be considered with caution. This map explores the parameter space in two dimensions, without taking into account potential correlations with the other parameters. As already discussed, if the best-fit estimate of the other parameters changes, the entire map might change as well. In order to give proper stability constraints on the orbital parameters and masses of the planets, we also performed a global stability analysis from the posterior distribution of the joint photometry + RV analysis. The only fixed parameter is the inclination of the outer planet, taken equal to the inclination of the inner planet (i.e., we explored the co-planar case, which is compatible with the outer planet non transiting). We selected a sample of 10000 solutions from that posterior. Each is numerically integrated over $20 \mathrm{kyr}$ using the same integrator set-up described in the previous section. The level of chaos is again computed with the NAFF indicator. Over the 10000 configurations, 8455 survived the entire $20 \mathrm{kyr}$ simulation; the others were unstable (escape or close encounter of two bodies). Imposing a more strict stability threshold by further removing all solutions with NAFF $>-5$ leaves us with a stable posterior of 7224 configurations.

In any case, no stringent constraint can be added on the orbital parameters and masses of the planets. With the sorting in NAFF as defined above, only a slight cut in the eccentricities is observed. The new median estimates and $1 \sigma$ confidence intervals are $e_{b}=0.162[0.098,0.228]$ and $e_{c}=0.129$ [0.065,0.205]. In particular, the global stability analysis does not allow us to further constrain the planetary masses (again assuming co-planar orbits).

\subsection{Atmospheric characterization}

TOI $1062 \mathrm{~b}$ poses an interesting target for atmospheric characterization given its equilibrium temperature of $\sim 1000 \mathrm{~K}$. We expect a high-metallicity atmosphere for a planet with such a small radius and mass, as a result of the strong stellar irradiation received which would result in a significant loss of the $\mathrm{H}_{2} / \mathrm{He}$ envelope. However, such a target is an excellent laboratory to study carbon chemistry at high temperature given its high expected metallicity. Beyond metallicities of $\gtrsim 300 \times$ solar, the primary carbon bearing species in the atmosphere transitions from being $\mathrm{CH}_{4} / \mathrm{CO}$ to $\mathrm{CO}_{2}$ in thermochemical equilibrium (e.g., Moses et al. 2013). This planet is thus an ideal target to explore the $\mathrm{CO}, \mathrm{CH}_{4}$, and $\mathrm{CO}_{2}$ chemical stability boundary which occurs at $\sim 800 \mathrm{~K}$ in photospheric conditions at such high metallicities. The mean molecular weight for such an atmosphere at $\sim 300 \times$ metallicity is expected to be $\sim 4 \mathrm{~g} \mathrm{~mol}^{-1}$, and hence significant features are still present in transmission spectra compared to a solar metallicity atmosphere (at $\sim 2.35 \mathrm{~g} \mathrm{~mol}^{-1}$ ). Using the transmission spectroscopy metric (TSM) defined in Kempton et al. (2018), we determine that TOI 1062b has a TSM value of 31.8 assuming a mean molecular mass of $2.3 \mathrm{~g} \mathrm{~mol}^{-1}$ and 18.3 assuming $4 \mathrm{~g} \mathrm{~mol}^{-1}$. The lower TSM value for the higher mean molecular weight is due to the reduced atmospheric scale height of a heavier atmosphere. This value is comparable to targets such as Trappist-1f and several simulated TESS targets from Sullivan et al. (2015).

On the other hand, TOI 1062b may have completely lost all of its $\mathrm{H}_{2} / \mathrm{He}$ envelope which would result in an ultrahigh-metallicity secondary atmosphere. At metallicities $\gtrsim 3000 \times$ solar, the mean molecular weight of the heavier species such as $\mathrm{CO}_{2}$ and $\mathrm{H}_{2} \mathrm{O}$ now completely dominate due to the lack of significant $\mathrm{H}_{2}$ or He. Therefore, the mean molecular weight is likely to be $>18 \mathrm{~g} / \mathrm{mol}$, and thus the scale height of spectral features in the transmission spectra are significantly reduced by a factor of $\gtrsim 7 \times$ over a solar metallicity atmosphere. This reduces the TSM value for such an atmosphere to $\lesssim 5$, making constraints on the abundances difficult. However, observations of the terminator may still be able to place a lower limit on the molecular weight, and thus the metallicity. They would also provide a direct contrast to planets such as 55 Cancri e which also indicate a high mean molecular weight atmosphere (Jindal et al. 2020). In addition, secondary eclipse spectroscopy for such a cool target is challenging, but has been achieved for GJ436b (Stevenson et al. 2010). However, for the case of TOI 1062b, the host star is a G-type and thus will result in a very weak planet-star flux contrast in emission spectroscopy.

\section{Conclusions}

We present the discovery of two new planets from the TESS mission in the TOI 1062 system. The analysis is based on 2 min cadence TESS observations from four sectors, ground-based follow-up from LCOGT, and RV data from the HARPS spectrograph. High-resolution imaging from Zorro speckle imager rules out the presence of nearby companions and potential nearby eclipsing binaries. We find that the host star is rotating with a period of 21.9 days, and also note the existence of a stellar spot whose lifetime is similar to the stellar rotational period.

TOI $1062 \mathrm{~b}$ is expected to be a mini-Neptune with a period of 4.11 days, radius of $2.27 \pm 0.1 R_{\oplus}$, and mass of $10.2 \pm 0.8 M_{\oplus}$. Internal structure models indicate that TOI $1062 \mathrm{~b}$ is expected to be composed of significant iron core and silicate mantle, accounting for nearly $40 \%$ of the planetary mass each, and that it is expected to have a small volatile envelope of $0.35 \%$ of the mass at most. TOI 1062c, which is not transiting, is found in the HARPS RV data. We find that its minimum mass is inferred to be $9.8 \pm 1.2 M_{\oplus}$, and that it is close to a $2: 1$ motion resonance with its inner companion, with a period of 7.97 days. The position of TOI $1062 \mathrm{~b}$ with respect to the radius valley and its high equilibrium temperature of $\sim 1000 \mathrm{~K}$ make it an interesting candidate for atmospheric characterization. The strong stellar irradiation may result in a significant loss of the $\mathrm{H}-\mathrm{He}$ envelope leaving a high-metallicity atmosphere that can be an excellent laboratory to study carbon chemistry at high temperature.

Acknowledgements. This work has been in particular carried out in the frame of the National Centre for Competence in Research 'PlanetS' supported by SNSF. D.J.A. acknowledges support from the STFC via an Ernest Rutherford Fellowship (ST/R00384X/1). This work was supported by FCT - Fundação para a Ciência e a Tecnologia through national funds and by FEDER through COMPETE2020 - Programa Operacional Competitividade e Internacionalização by these grants: UID/FIS/04434/2019; UIDB/04434/2020; UIDP/04434/2020; PTDC/FIS-AST/32113/2017 and POCI-01-0145-FEDER032113; PTDC/FIS-AST/28953/2017 and POCI-01-0145-FEDER-028953. V.A. and E.D.M acknowledge the support from FCT through Investigador FCT contracts nr. IF/00650/2015/CP1273/CT0001, IF/00849/2015/CP1273/CT0003, respectively. C.D. acknowledges support from the Swiss National Science Foundation under grant PZ00P2_174028. Siddharth Gandhi acknowledges support from the UK Science and Technology Facilities Council (STFC) research grant 
ST/S000631/1. Resources supporting this work were provided by the NASA High-End Computing (HEC) Program through the NASA Advanced Supercomputing (NAS) Division at Ames Research Center for the production of the SPOC data products. We acknowledge the use of public TESS Alert data from pipelines at the TESS Science Office and at the TESS Science Processing Operations Center. This research has been partly funded by the Spanish State Research Agency (AEI) Projects No. ESP2017-87676-C5-1-R and No. MDM-2017-0737 Unidad de Excelencia "María de Maeztu"- Centro de Astrobiología (INTA-CSIC). S.G.S acknowledges the support from FCT through Investigador FCT contract nr. CEECIND/00826/2018 and POPH/FSE (EC) H.P.O. acknowledges that this work has been carried out within the framework of the NCCR PlanetS supported by the Swiss National Science Foundation. S.H. acknowledges support by the fellowships PD/BD/128119/2016 funded by FCT (Portugal). X.D is grateful to The Branco Weiss Fellowship-Society in Science for its financial support. This project has received funding from the European Research Council (ERC) under the European Union's Horizon 2020 research and innovation programme (grant agreement No 851555/SCORE. A.O acknowledges support from an STFC studentship. S.H acknowledges CNES funding through the grant 837319.This work was supported by FCT through national funds (PTDC/FIS-AST/28953/2017) and by FEDER - Fundo Europeu de Desenvolvimento Regional through COMPETE2020 - Programa Operacional Competitividade e Internacionalização (POCI-01-0145-FEDER-028953) and through national funds (PIDDAC) by the grant UID/FIS/04434/2019. Funding for the TESS mission is provided by NASA's Science Mission directorate. This work makes use of observations from the LCOGT network.

\section{References}

Adibekyan, V. Z., Sousa, S. G., Santos, N. C., et al. 2012, A\&A, 545, A32 Akeson, R. L., Chen, X., Ciardi, D., et al. 2013, PASP, 125, 989

Alibert, Y., Mordasini, C., Benz, W., \& Naef, D. 2010, EAS Pub. Ser., 42, 209

Alibert, Y., Thiabaud, A., Mzarboeuf, U., et al. 2015, AAS/Div. Extreme Sol. Syst. Abs., 47, 115.02

Anderson, J. D., Esposito, P. B., Martin, W., Thornton, C. L., \& Muhleman, D. O. 1975, ApJ, 200, 221

Armstrong, D. J., Lopez, T. A., Adibekyan, V., et al. 2020, Nature, 583, 39

Baluev, R. V. 2008, MNRAS, 385, 1279

Baranne, A., Queloz, D., Mayor, M., et al. 1996, A\&AS, 119, 373

Beaugé, C., \& Nesvorný, D. 2013, ApJ, 763, 12

Bertran de Lis, S., Delgado Mena, E., Adibekyan, V. Z., Santos, N. C., \& Sousa, S. G. 2015, A\&A, 576, A89

Borucki, W. J., Koch, D., \& Kepler Science Team. 2010, AAS/Div. Planet. Sci. Meeting Abs., 42, 47.03

Brown, T. M., Baliber, N., Bianco, F. B., et al. 2013, PASP, 125, 1031

Buchner, J., Georgakakis, A., Nandra, K., et al. 2014, A\&A, 564, A125

Chabrier, G., Mazevet, S., \& Soubiran, F. 2019, ApJ, 872, 51

Collins, K. A., Kielkopf, J. F., Stassun, K. G., \& Hessman, F. V. 2017, AJ, 153, 77

Connolly, J. A. D. 2009, Geochem. Geophys. Geosyst., 10, Q10014

Delgado Mena, E., Israelian, G., González Hernández, J. I., et al. 2010, ApJ, 725, 2349

Delgado Mena, E., Tsantaki, M., Adibekyan, V. Z., et al. 2017, A\&A, 606, A94

Delgado Mena, E., Moya, A., Adibekyan, V., et al. 2019, A\&A, 624, A78

Delisle, J. B., Hara, N., \& Ségransan, D. 2020, A\&A, 638, A95

Espinoza, N. 2018, Res. Notes Am. Astron. Soc., 2, 209

Espinoza, N., \& Jordán, A. 2015, MNRAS, 450, 1879

Espinoza, N., Kossakowski, D., \& Brahm, R. 2019, MNRAS, 490, 2262

Feroz, F., \& Hobson, M. P. 2008, MNRAS, 384, 449

Findeisen, K., Hillenbrand, L., \& Soderblom, D. 2011, AJ, 142, 23

Foreman-Mackey, D., Agol, E., Ambikasaran, S., \& Angus, R. 2017, AJ, 154, 220

Fulton, B. J., Petigura, E. A., Howard, A. W., et al. 2017, AJ, 154, 109

Fulton, B. J., Petigura, E. A., Blunt, S., \& Sinukoff, E. 2018, PASP, 130, 044504

Gaia Collaboration (Brown, A. G. A., et al.) 2018, A\&A, 616, A1

Ginzburg, S., Schlichting, H. E., \& Sari, R. 2018, MNRAS, 476, 759

Guerrero, N. 2020, Am. Astron. Soc. Meeting Abs., 235, 327.03

Guillot, T. 2010, A\&A, 520, A27

Gupta, A., \& Schlichting, H. E. 2019, MNRAS, 487, 24

Hakim, K., Rivoldini, A., Van Hoolst, T., et al. 2018, Icarus, 313, 61

Haldemann, J., Alibert, Y., Mordasini, C., \& Benz, W. 2020, A\&A, 643, A105

Hara, N. C., Boué, G., Laskar, J., \& Correia, A. C. M. 2017, MNRAS, 464, 1220

Hara, N. C., Bouchy, F., Stalport, M., et al. 2020, A\&A, 636, L6

Høg, E., Fabricius, C., Makarov, V. V., et al. 2000, A\&A, 355, L27

Howell, S. B., Everett, M. E., Sherry, W., Horch, E., \& Ciardi, D. R. 2011, AJ, 142,19

Howell, S. B., Sobeck, C., Haas, M., et al. 2014, PASP, 126, 398
Jenkins, J. M., Twicken, J. D., McCauliff, S., et al. 2016, Proc. SPIE, 9913, 99133E

Jenkins, J. S., Díaz, M. R., Kurtovic, N. T., et al. 2020, Nat. Astron., 4, 1148 Jensen, E. 2013, Astrophysics Source Code Library [record ascl: 1306.007] Jindal, A., de Mooij, E. J. W., Jayawardhana, R., et al. 2020, AJ, 160, 101 Kempton, E. M. R., Bean, J. L., Louie, D. R., et al. 2018, PASP, 130, 114401 Kipping, D. M. 2013, MNRAS, 435, 2152

Kreidberg, L. 2015, PASP, 127, 1161

Kurucz, R. L. 1993, SYNTHE Spectrum Synthesis Programs and Line Data (Cambridge: Smithsonian Astrophysical Observatory)

Laskar, J. 1993, Phys. D Nonlinear Phenom., 67, 257

Laskar, J., Froeschlé, C., \& Celletti, A. 1992, Phys. D Nonlinear Phenom., 56, 253

Li, J., Tenenbaum, P., Twicken, J. D., et al. 2019, PASP, 131, 024506

Lopez, E., \& Fortney, J. J. 2013, AAS/Div. Planet. Sci. Meeting Abs., 45, 200.08

Lucy, L. B., \& Sweeney, M. A. 1971, AJ, 76, 544

Mamajek, E. E., \& Hillenbrand, L. A. 2008, ApJ, 687, 1264

Mayor, M., Pepe, F., Queloz, D., et al. 2003, The Messenger, 114, 20

Mazeh, T., Holczer, T., \& Faigler, S. 2016, A\&A, 589, A75

Moses, J. I., Line, M. R., Visscher, C., et al. 2013, ApJ, 777, 34

Nielsen, L. D., Gandolfi, D., Armstrong, D. J., et al. 2020, MNRAS, 492, 5399

Otegi, J. F., Bouchy, F., \& Helled, R. 2020a, A\&A, 634, A43

Otegi, J. F., Dorn, C., Helled, R., et al. 2020b, A\&A, 640, A135

Owen, J. E., \& Jackson, A. P. 2012, MNRAS, 425, 2931

Pepe, F., Mayor, M., Rupprecht, G., et al. 2002, The Messenger, 110, 9

Plotnykov, M., \& Valencia, D. 2020, MNRAS, 499, 932

Rein, H., \& Liu, S. F. 2012, A\&A, 537, A128

Rein, H., \& Spiegel, D. S. 2015, MNRAS, 446, 1424

Ricker, G. R., Winn, J. N., Vanderspek, R., et al. 2015, J. Astron. Teles. Instrum. Syst., 1, 014003

Santos, N. C., Israelian, G., \& Mayor, M. 2004, A\&A, 415, 1153

Santos, N. C., Sousa, S. G., Mortier, A., et al. 2013, A\&A, 556, A150

Scott, N. J. 2019, AAS/Div. Extreme Sol. Syst. Abs., 51, 330.15

Skrutskie, M. F., Cutri, R. M., Stiening, R., et al. 2006, AJ, 131, 1163

Smith, J. C., Stumpe, M. C., Van Cleve, J. E., et al. 2012, PASP, 124, 1000

Sneden, C. A. 1973, PhD thesis, The University of Texas at Austin, USA

Sousa, S. G. 2014, Determination of Atmospheric Parameters of B-, A-, F- and G-

Type Stars, GeoPlanet: Earth and Planetary Sciences (Springer International Publishing (Cham)), eds. E. Niemczura, B. Smalley \& W. Pych, 297

Sousa, S. G., Santos, N. C., Adibekyan, V., Delgado-Mena, E., \& Israelian, G. 2015, A\&A, 577, A67

Speagle, J. S. 2020, MNRAS, 493, 3132

Stassun, K. G., \& Torres, G. 2016, AJ, 152, 180

Stassun, K. G., \& Torres, G. 2018, ApJ, 862, 61

Stassun, K. G., Collins, K. A., \& Gaudi, B. S. 2017, AJ, 153, 136

Stassun, K. G., Corsaro, E., Pepper, J. A., \& Gaudi, B. S. 2018, AJ, 155, 22

Stevenson, K. B., Harrington, J., Nymeyer, S., et al. 2010, Nature, 464, 1161

Stixrude, L., \& Lithgow-Bertelloni, C. 2011, Geophys. J. Int., 184, 1180

Sullivan, P. W., Winn, J. N., Berta-Thompson, Z. K., et al. 2015, ApJ, 809, 77

Szabó, G. M., \& Kiss, L. L. 2011, ApJ, 727, L44

Tamayo, D., Rein, H., Shi, P., \& Hernandez, D. M. 2019, MNRAS, 491, 2885

Torres, G., Andersen, J., \& Giménez, A. 2010, A\&ARv, 18, 67

Twicken, J. D., Chandrasekaran, H., Jenkins, J. M., et al. 2010, Proc. SPIE, 7740, $77401 \mathrm{U}$

Twicken, J. D., Catanzarite, J. H., Clarke, B. D., et al. 2018, PASP, 130, 064502

Venturini, J., \& Helled, R. 2017, ApJ, 848, 95

Wang, H. S., Liu, F., Ireland, T. R., et al. 2019, MNRAS, 482, 2222

Wright, E. L., Eisenhardt, P. R. M., Mainzer, A. K. et al. 2010, AJ, 140, 1868

1 Observatoire Astronomique de l'Université de Genève, $51 \mathrm{Ch}$. des Maillettes, Sauverny, 1290 Versoix, Switzerland e-mail: jonfr17@gmail.com

2 Institute for Computational Science, University of Zurich, Winterthurerstr. 190, 8057 Zurich, Switzerland

3 Center for Astrobiology (CAB, INTA-CSIC), Dept. de Astrofísica, ESAC campus 28692 Villanueva de la Cañada (Madrid), Spain

4 Department of Physics and Kavli Institute for Astrophysics and Space Research, Massachusetts Institute of Technology, Cambridge, MA 02139, USA

5 Department of Earth, Atmospheric and Planetary Sciences, Massachusetts Institute of Technology, Cambridge, MA 02139, USA 
${ }^{6}$ Department of Aeronautics and Astronautics, MIT, 77 Massachusetts Avenue, Cambridge, MA 02139, USA

7 Center for Astrophysics, Harvard \& Smithsonian, 60 Garden Street, Cambridge, MA 02138, USA

8 American Association of Variable Star Observers, 49 Bay State Road, Cambridge, MA 02138, USA

9 Patashnick Voorheesville Observatory, Voorheesville, NY 12186, USA

10 Tsinghua International School, Beijing 100084, PR China

11 Department of Astrophysical Sciences, Princeton University, NJ 08544, USA

12 NASA Ames Research Center, Moffett Field, CA 94035, USA

13 Vanderbilt University, Department of Physics \& Astronomy, Nashville, TN 37235, USA

${ }^{14}$ Astrophysics Science Division, NASA Goddard Space Flight Center, Greenbelt, MD 20771, USA

15 Department of Astronomy, The University of Texas at Austin, Austin, TX 78712, USA

16 Caltech/IPAC-NExScI, M/S 100-22, 1200 E California Blvd, Pasadena, CA 91125, USA

17 Instituto de Astrofísica e Ciências do Espaço, Universidade do Porto, CAUP, Rua das Estrelas, 4150-762 Porto, Portugal
${ }^{18}$ Departamento de Física e Astronomia, Faculdade de Ciências, Universidade do Porto, Rua do Campo Alegre, Porto, Portugal

19 Department of Physics, University of Warwick, Coventry CV4 7AL, UK

20 Centre for Exoplanets and Habitability, University of Warwick, Gibbet Hill Road, Coventry CV4 7AL, UK

${ }^{21}$ SETI Institute, Mountain View, CA 94043, USA

${ }^{22}$ Space Telescope Science Institute, 3700 San Martin Drive, Baltimore, MD 21218, USA

${ }^{23}$ NCCR/PlanetS, Centre for Space \& Habitability, University of Bern, Bern, Switzerland

${ }^{24}$ Department of Physics and Kavli Institute for Astrophysics and Space Research, Massachusetts Institute of Technology, Cambridge, MA 02139, USA

25 Leiden Observatory, University of Leiden, PO Box 9513, 2300 RA Leiden, The Netherlands

${ }^{26}$ Department of Earth and Space Sciences, Chalmers University of Technology, Onsala Space Observatory, 439 92, Onsala, Sweden

27 Aix Marseille Univ, CNRS, CNES, LAM, Marseille, France

${ }^{28}$ European Southern Observatory, Alonso de Córdova 3107, Vitacura, Santiago, Chile 


\section{Appendix A: HARPS spectroscopy}

Table A.1. HARPS spectroscopy obtained between 31 August 2019 and 11 November 2019.

\begin{tabular}{|c|c|c|c|c|c|c|}
\hline $\begin{array}{l}\text { Time } \\
{[\mathrm{BJD}-2400000]}\end{array}$ & $\begin{array}{l}\mathrm{RV} \\
\qquad\left[\mathrm{m} \mathrm{s}^{-1}\right.\end{array}$ & ${ }_{1}^{\sigma_{\mathrm{RV}}}$ & $S_{\mathrm{MW}}$ & $\sigma_{\mathrm{S}}$ & $\begin{array}{l}F W H M \\
\qquad\left[\mathrm{~m} \mathrm{~s}^{-1}\right.\end{array}$ & $\sigma_{\mathrm{FWHM}}$ \\
\hline 58726.77 & -4.63 & 1.19 & 0.2308 & 0.0035 & 6521.73 & 9.7 \\
\hline 58726.83 & -3.58 & 1.39 & 0.2205 & 0.0051 & 6516.21 & 15.2 \\
\hline 58727.78 & -4.91 & 1.19 & 0.2218 & 0.0032 & 6514.78 & 9.6 \\
\hline 58727.86 & -2.92 & 1.19 & 0.2223 & 0.0035 & 6520.8 & 10.4 \\
\hline 58728.71 & -4.13 & 1.11 & 0.2305 & 0.0026 & 6511.39 & 7.3 \\
\hline 58728.76 & -3.05 & 1.03 & 0.2284 & 0.0021 & 6513.01 & 5.9 \\
\hline 58729.76 & -5.6 & 1.16 & 0.2246 & 0.0031 & 6511.48 & 9.0 \\
\hline 58729.86 & -5.54 & 1.1 & 0.226 & 0.0029 & 6511.24 & 8.2 \\
\hline 58730.74 & -0.72 & 1.49 & 0.2145 & 0.0051 & 6510.23 & 16.0 \\
\hline 58730.82 & 1.18 & 1.49 & 0.2139 & 0.0055 & 6515.79 & 17.1 \\
\hline 58744.77 & -1.12 & 1.68 & 0.2143 & 0.0068 & 6522.73 & 21.2 \\
\hline 58745.74 & -16.3 & 3.27 & 0.2023 & 0.0179 & 6534.68 & 61.2 \\
\hline 58746.69 & 0.09 & 1.54 & 0.2148 & 0.0055 & 6517.99 & 17.2 \\
\hline 58747.68 & 6.78 & 2.19 & 0.2099 & 0.01 & 6505.19 & 30.9 \\
\hline 58747.7 & 4.5 & 1.73 & 0.2089 & 0.0068 & 6513.01 & 22.0 \\
\hline 58748.75 & 7.97 & 1.56 & 0.2267 & 0.0056 & 6507.91 & 16.2 \\
\hline 58750.76 & -2.32 & 1.4 & 0.2289 & 0.0046 & 6522.1 & 13.1 \\
\hline 58760.76 & -1.13 & 1.79 & 0.1976 & 0.008 & 6507.77 & 28.3 \\
\hline 58760.85 & 1.43 & 1.34 & 0.2064 & 0.005 & 6511.14 & 16.5 \\
\hline 58761.72 & -1.0 & 1.22 & 0.2259 & 0.0037 & 6515.05 & 10.7 \\
\hline 58762.71 & -2.72 & 1.33 & 0.2152 & 0.0042 & 6512.07 & 13.1 \\
\hline 58763.78 & 3.58 & 1.13 & 0.2196 & 0.003 & 6506.89 & 9.1 \\
\hline 58764.67 & 0.32 & 1.21 & 0.219 & 0.0031 & 6509.88 & 9.5 \\
\hline 58765.71 & -43.31 & 4.43 & 0.23 & 0.0271 & 6569.43 & 76.2 \\
\hline 58766.69 & -13.89 & 1.11 & 0.2193 & 0.0026 & 6502.88 & 7.7 \\
\hline 58773.73 & -2.55 & 1.66 & 0.202 & 0.0069 & 6513.39 & 23.6 \\
\hline 58774.69 & -10.79 & 1.31 & 0.2135 & 0.0039 & 6507.43 & 12.3 \\
\hline 58774.8 & -13.66 & 1.57 & 0.1942 & 0.0062 & 6509.0 & 22.5 \\
\hline 58775.67 & -10.02 & 1.31 & 0.2203 & 0.0042 & 6507.57 & 12.5 \\
\hline 58775.81 & -11.1 & 1.89 & 0.2004 & 0.0084 & 6519.12 & 29.1 \\
\hline 58776.74 & -0.04 & 2.33 & 0.2159 & 0.0118 & 6514.25 & 36.6 \\
\hline 58776.8 & -8.42 & 2.18 & 0.2083 & 0.011 & 6509.26 & 36.0 \\
\hline 58777.66 & 1.5 & 1.65 & 0.2212 & 0.0065 & 6519.42 & 19.3 \\
\hline 58777.78 & -4.05 & 2.79 & 0.2323 & 0.015 & 6512.9 & 41.6 \\
\hline 58791.6 & -8.57 & 1.61 & 0.2249 & 0.0061 & 6511.53 & 17.7 \\
\hline 58791.78 & -6.63 & 1.85 & 0.1908 & 0.0084 & 6506.83 & 31.5 \\
\hline 58792.62 & -1.06 & 1.31 & 0.2249 & 0.004 & 6506.84 & 11.6 \\
\hline 58792.76 & -2.15 & 1.28 & 0.2163 & 0.0044 & 6506.41 & 13.5 \\
\hline 58793.62 & 5.46 & 1.26 & 0.2296 & 0.0033 & 6508.11 & 9.3 \\
\hline 58793.78 & 6.2 & 1.22 & 0.2152 & 0.004 & 6510.24 & 12.5 \\
\hline 58794.6 & 2.03 & 1.36 & 0.2122 & 0.0041 & 6510.28 & 13.1 \\
\hline 58794.71 & 2.13 & 1.3 & 0.2181 & 0.004 & 6507.05 & 12.2 \\
\hline 58795.59 & -2.05 & 1.53 & 0.2051 & 0.0046 & 6506.22 & 15.3 \\
\hline 58795.72 & -1.34 & 1.28 & 0.213 & 0.0037 & 6506.98 & 11.6 \\
\hline 58796.6 & -1.28 & 1.17 & 0.2217 & 0.0029 & 6505.65 & 8.7 \\
\hline 58796.73 & -0.69 & 1.2 & 0.2181 & 0.0034 & 6502.47 & 10.3 \\
\hline 58797.6 & -2.84 & 1.31 & 0.2107 & 0.0036 & 6503.46 & 11.6 \\
\hline 58797.73 & -0.47 & 1.41 & 0.2095 & 0.0046 & 6507.76 & 15.0 \\
\hline 58798.67 & -5.87 & 1.21 & 0.2189 & 0.003 & 6503.02 & 9.2 \\
\hline 58799.75 & -16.63 & 1.53 & 0.203 & 0.0058 & 6509.75 & 19.8 \\
\hline 58799.86 & -23.92 & 1.56 & 0.1928 & 0.0064 & 6525.7 & 23.5 \\
\hline
\end{tabular}

Table A.2. HARPS spectroscopy obtained between 20 November 2019 and 17 January 2020.

\begin{tabular}{|c|c|c|c|c|c|c|}
\hline $\begin{array}{l}\text { Time } \\
\text { [BJD - } 2400000]\end{array}$ & $\begin{array}{l}\mathrm{RV} \\
\qquad \mathrm{m} \mathrm{s}^{-1}\end{array}$ & $\begin{array}{c}\sigma_{\mathrm{RV}} \\
1]\end{array}$ & $S_{\mathrm{MW}}$ & $\sigma_{S}$ & $\begin{array}{l}F W H M \\
\qquad\left[\mathrm{~m} \mathrm{~s}^{-1}\right. \\
\end{array}$ & $\begin{array}{l}\sigma_{\mathrm{FWHM}} \\
\left.{ }^{1}\right]\end{array}$ \\
\hline 58808.67 & -0.17 & 1.29 & 0.2389 & 0.0041 & 6538.41 & 10.9 \\
\hline 58810.65 & 4.55 & 1.29 & 0.2362 & 0.0038 & 6530.36 & 10.4 \\
\hline 58811.71 & -0.94 & 1.27 & 0.228 & 0.004 & 6527.1 & 11.3 \\
\hline 58816.73 & -7.0 & 1.1 & 0.2152 & 0.003 & 6504.0 & 9.4 \\
\hline 58819.7 & -6.58 & 1.4 & 0.2183 & 0.0044 & 6501.38 & 13.3 \\
\hline 58821.75 & -3.34 & 1.41 & 0.2111 & 0.0047 & 6496.4 & 14.9 \\
\hline 58823.73 & -12.12 & 1.5 & 0.2006 & 0.0053 & 6497.09 & 18.5 \\
\hline 58830.59 & -3.48 & 0.98 & 0.2136 & 0.002 & 6495.06 & 6.2 \\
\hline 58830.69 & -4.1 & 1.05 & 0.2117 & 0.0027 & 6496.99 & 8.7 \\
\hline 58831.54 & -7.06 & 1.02 & 0.2134 & 0.0019 & 6490.0 & 6.1 \\
\hline 58831.67 & -9.5 & 1.15 & 0.2176 & 0.0033 & 6498.19 & 9.9 \\
\hline 58832.55 & -10.45 & 1.63 & 0.2077 & 0.0057 & 6500.63 & 18.7 \\
\hline 58832.66 & -9.7 & 1.75 & 0.2083 & 0.0069 & 6509.54 & 20.8 \\
\hline 58833.61 & -1.92 & 2.39 & 0.1895 & 0.0109 & 6499.71 & 41.1 \\
\hline 58834.58 & 9.53 & 1.31 & 0.2155 & 0.0039 & 6500.35 & 12.2 \\
\hline 58834.68 & 9.52 & 1.28 & 0.2154 & 0.0042 & 6505.94 & 12.9 \\
\hline 58835.58 & 11.45 & 1.06 & 0.2275 & 0.0022 & 6514.18 & 6.4 \\
\hline 58835.69 & 8.87 & 1.2 & 0.2284 & 0.0038 & 6507.52 & 10.7 \\
\hline 58836.55 & 7.75 & 1.05 & 0.2285 & 0.0022 & 6518.42 & 6.2 \\
\hline 58836.69 & 7.74 & 1.13 & 0.2274 & 0.0031 & 6516.43 & 8.8 \\
\hline 58837.57 & 10.35 & 1.23 & 0.2329 & 0.0034 & 6519.22 & 9.4 \\
\hline 58837.69 & 8.19 & 1.42 & 0.2273 & 0.0051 & 6528.12 & 14.6 \\
\hline 58838.66 & 12.94 & 1.33 & 0.2383 & 0.0044 & 6522.37 & 11.8 \\
\hline 58839.55 & 9.99 & 1.12 & 0.2455 & 0.0025 & 6531.33 & 6.4 \\
\hline 58839.61 & 9.62 & 1.14 & 0.2524 & 0.0029 & 6533.14 & 7.1 \\
\hline 58840.63 & 4.6 & 1.09 & 0.2506 & 0.003 & 6536.72 & 7.4 \\
\hline 58841.68 & 9.26 & 1.15 & 0.243 & 0.0035 & 6536.59 & 9.1 \\
\hline 58842.63 & 14.97 & 1.21 & 0.2412 & 0.0038 & 6537.23 & 9.9 \\
\hline 58843.56 & 10.7 & 1.03 & 0.2515 & 0.0021 & 6535.97 & 5.2 \\
\hline 58844.59 & 0.13 & 1.16 & 0.2475 & 0.0031 & 6532.33 & 7.8 \\
\hline 58845.67 & -4.45 & 1.34 & 0.2389 & 0.0046 & 6523.74 & 12.3 \\
\hline 58847.65 & -8.6 & 1.22 & 0.2252 & 0.0038 & 6512.18 & 11.1 \\
\hline 58848.6 & -12.47 & 1.39 & 0.2146 & 0.0046 & 6510.43 & 14.4 \\
\hline 58848.61 & -13.08 & 1.44 & 0.2291 & 0.0047 & 6510.57 & 13.4 \\
\hline 58849.64 & -9.39 & 1.23 & 0.219 & 0.0038 & 6507.37 & 11.6 \\
\hline 58850.58 & 0.44 & 1.16 & 0.2219 & 0.0029 & 6501.77 & 8.4 \\
\hline 58852.57 & -3.63 & 1.03 & 0.22 & 0.0024 & 6503.71 & 7.1 \\
\hline 58853.61 & -6.25 & 1.18 & 0.2178 & 0.0034 & 6498.99 & 9.7 \\
\hline 58855.64 & 2.87 & 2.48 & 0.1971 & 0.0119 & 6509.76 & 42.5 \\
\hline 58865.55 & -5.45 & 1.2 & 0.2276 & 0.0035 & 6511.98 & 9.9 \\
\hline
\end{tabular}

